Accepted for publication in ApJ.

Preprint typeset using $\mathrm{LAT}_{\mathrm{E}} \mathrm{X}$ style emulateapj v. 08/13/06

\title{
EQUAL- AND UNEQUAL-MASS MERGERS OF DISK AND ELLIPTICAL GALAXIES WITH BLACK HOLES
}

\author{
Peter H. Johansson ${ }^{1}$, Thorsten NaAB ${ }^{1}$, Andreas Burkert ${ }^{1}$ \\ Accepted for publication in ApJ.
}

\begin{abstract}
We present binary galaxy merger simulations with varying mass ratios and different progenitor morphologies. The simulations include mergers of gas-rich disks (Sp-Sp), of early-type galaxies and disks (E-Sp, mixed mergers), and mergers of early-type galaxies (E-E, dry mergers). We follow the dynamics of gas, stars and dark matter, and include radiative cooling, star formation and black hole $(\mathrm{BH})$ accretion and the associated feedback processes as in Springel et al. We study the mass assembly of the BHs and discuss technical issues of the implemented model in detail. For Sp-Sp mergers, the peak star formation rate and $\mathrm{BH}$ accretion rate decrease and the growth timescales of the central black holes and newly formed stars increase with higher progenitor mass ratios. The peak $\mathrm{BH}$ accretion rate typically occurs shortly after the time of $\mathrm{BH}$ merging for low progenitor mass ratios (e.g. 3:1 and lower), whereas for higher progenitor mass ratios there is no clear correlation between the peak $\mathrm{BH}$ accretion rate and $\mathrm{BH}$ merging time. The termination of star formation by $\mathrm{BH}$ feedback in disk mergers is significantly less important for higher progenitor mass ratios (e.g. 3:1 and higher). In addition, the inclusion of BH feedback suppresses efficiently star formation in dry E-E mergers and mixed E-Sp mergers. All merger remnants, independent of their progenitors, follow the observed relations between the central $\mathrm{BH}$ mass and the stellar velocity dispersion $\left(M_{\mathrm{BH}}-\sigma\right)$, the bulge mass $\left(M_{\mathrm{BH}}-M_{*}\right)$ and the bulge binding energy $\left(M_{\mathrm{BH}}-M_{*} \sigma^{2}\right)$, with the dominant source of scatter arising from variations in the initial gas mass fraction. The normalizations for all relations and the simulated slope of the $M_{\mathrm{BH}}-\sigma$ and $M_{\mathrm{BH}}-M_{*} \sigma^{2}$ relations are in good agreement with the observations, whereas the simulated slope of the $M_{\mathrm{BH}}-M_{*}$ relation is slightly steeper compared to the observations. This indicates that the simple $\mathrm{BH}$ feedback model self-regulates the mass growth of the $\mathrm{BHs}$ in accordance with the observed relations for a very wide range of merger progenitors.
\end{abstract}

Subject headings: galaxies: interaction-galaxies: active galaxies: evolution - galaxies: formation methods: numerical

\section{INTRODUCTION}

Observations in the last decade have shown that supermassive black holes $(\mathrm{BH})$ reside in the centers of most if not all galaxies with spheroids (e.g. Kormendy \& Richstone 1995; Richstone et al. 1998). The properties of these black holes and their host galaxies are correlated in a number of ways, e.g. the correlation of the $\mathrm{BH}$ mass and the bulge velocity dispersion as expressed in the $M_{\mathrm{BH}}-\sigma$ relation (e.g. Ferrarese \& Merritt 2000; Gebhardt et al. 2000; Tremaine et al. 2002), the relation between $\mathrm{BH}$ mass and the bulge stellar mass, $M_{\mathrm{BH}}-M_{\text {bulge }}$ (e.g. Magorrian et al. 1998; Marconi \& Hunt 2003; Häring \& Rix 2004) and the relation between $\mathrm{BH}$ mass and the bulge binding energy, $M_{\mathrm{BH}}-M_{\text {bulge }} \sigma^{2}$ (e.g. Aller \& Richstone 2007; Barwav \& Kembhavi 2007). These observed relations indicate that supermassive BHs and the stellar spheroids of their host galaxies do not form independently of each other.

There exists several theoretical explanations for the observed correlation between galaxy properties and the black hole mass, including physical mechanisms such as viscous disk accretion and star formation (Burkert \& Silk 2001), gas or dark matter collapse (Adams et al. 2001), dissipationless merging (Ciotti \& van Albada 2001) and the merging and migration of massive stellar clumps

\footnotetext{
Electronic address: pjohan@usm.lmu.de

${ }^{1}$ University Observatory Munich, Scheinerstr. 1, D-81679 Munich, Germany
}

and their associated intermediate-mass $\mathrm{BHs}$ to the galactic centers (Bournaud et al. 2007a; Elmegreen et al. 2008). The majority of the models rely on some form of self-regulated $\mathrm{BH}$ mass growth, in which gas is fed to the central black hole until the black hole releases sufficient energy to unbind the gas and blow it away in momentum- or pressure-driven winds (e.g. Ciotti \& Ostriker 1997; Silk \& Rees 1998; Fabian 1999; Murray et al. 2005; Ciotti \& Ostriker 2007).

The first theoretical studies of the co-evolution of black holes and galaxies were carried out using semi-analytic modeling (SAM) techniques (e.q. Cattaneo et al. 1999; Kauffmann \& Haehnelt 2000; Wvithe \& Loeb 2003; Volonteri et al. 2003; Granato et al. 2004; Croton et al. 2006; Bower et al. 2006). In SAMs the evolution of the dark matter halos is followed with merger trees and the evolution of the galaxies and their embedded black holes is modeled with simple physical parameterizations. Most SAMs are based on the assumption that quasar activity is triggered by major galaxy mergers of gas-rich galaxies, in which large quantities of gas flow to the centers of galaxies (e.g. Barnes \& Hernquist 1996; Kazantzidis et al. 2005; Naab et al. 2006a; Hopkins et al. 2008b) producing starbursts and leading to rapid black hole growth (Hernquist 1989). During the mergers the dominant dynamical process is the violent relaxation (Lvnden-Bell 1967; Arad \& Johansson 2005) of the stellar and dark matter components.

This picture is supported by observations of local mergers that show large central gas concentrations, accompa- 
nied by strong starburst and AGN activity (Genzel et al. 1998), indicating BH growth. These so called ultraluminous infrared galaxies (ULIRGs) mostly originate from major mergers, typically with mass ratios below 3:1 (e.g. Dasyra et al. 2006a.c; Väisänen et al. 2008). The ULIRGs could eventually evolve into spheroidal galaxies (Hibbard \& Yun 1999; Genzel et al. 2001; Rothberg \& Joseph 2006; Dasvra et al. 2006b). In addition, nearby quasars (e.g. McLure et al. 1999; Canalizo \& Stockton 2001) are preferentially found in tidally disturbed objects, thus corroborating the important role of galaxy interactions and mergers for major black hole growth. Furthermore, an increasing number of recent infrared and X-ray surveys have completed the census of $\mathrm{BH}$ activity and mass growth by revealing a significant population of highly obscured AGNs at redshifts above $z \sim 1$ (Johansson et al. 2004; Houck et al. 2005; Barger et al. 2005).

A first, simple self-consistent method for including black hole growth and thermal feedback coupling to the surrounding gas in simulations of binary disk galaxy mergers was developed by Springel et al. (2005b). Based on this description hydrodynamical simulations of $\mathrm{BH}$ growth in galaxy mergers were used to reproduce the local $M_{\mathrm{BH}}-\sigma$ relation (Di Matteo et al. 2005), study the redshift evolution of this relation (Robertson et al. 2006c), provide an explanation of the red colors of massive ellipticals (Springel et al. 2005a), and describe the fundamental plane of elliptical galaxies (Robertson et al. 2006b). Using this black hole merger sample Hopkins et al. (2005, 2006) proposed a comprehensive picture of a unified merger-driven model for the origin of starbursts, quasars and their relation to galaxy spheroid formation. Recently, simulations of both individual galaxies (Khalatvan et al. 2008) and larger cosmological volumes (Sijacki et al. 2007; Di Matteo et al. 2008) with BH feedback starting directly from initial conditions appropriate for the $\Lambda$ CDM cosmology have been performed. Both cosmological studies assumed that seed black holes were present at high redshifts in forming halos and then followed their evolution self-consistently with hydrodynamical simulations. Di Matteo et al. (2008) employed the Springel et al. (2005b) black hole feedback prescription, whereas Sijacki et al. (2007) used in addition a predominantly mechanical feedback model for black holes in galaxy clusters with low accretion rates, in which AGN-driven bubbles are injected into a gaseous environment (Siiacki \& Springel 2006). The study by Di Matteo et al. (2005) showed that the $M_{\mathrm{BH}}-\sigma$ relation can be reproduced for equal-mass disk galaxy mergers. The cosmological studies of Sijacki et al. (2007) and Di Matteo et al. (2008) naturally include mergers with a range in mass ratios and morphologies, and both studies are able to find adequate fits to the observed $M_{\mathrm{BH}}-\sigma$ and $M_{\mathrm{BH}}-M_{*}$ relations at both high and low redshifts, where $M_{*}$ is defined as the total stellar mass within the effective radius. However, even the highest resolution cosmological simulations resolve spatial scales that are an order of magnitude larger than the typical spatial scales resolvable in high-resolution merger simulations $\left(\epsilon \sim 0.1 h^{-1} \mathrm{kpc}\right)$ and it is not obvious that low-resolution simulations that only resolve scales corresponding to the effective radii for typical galaxies are able to capture the essential physics related to $\mathrm{BH}$ feedback.
In a cosmological context unequal-mass mergers are more frequent than equal-mass mergers and the progenitors will have varying morphologies (Khochfar \& Burkert 2001; Khochfar \& Silk 2006). It has been shown that the mass ratio of disk mergers has a significant impact on the remnant properties. Equal-mass disk mergers predominantly result in slowly rotating systems with boxy or disky isophotes, whereas unequal-mass mergers lead to rotating, intrinsically anisotropic spheroids with disky isophotes (Barnes 1998; Naab et al. 1999; Cretton et al. 2001; Barnes 2002; Naab \& Burkert 2003; Bournaud et al. 2005; Burkert \& Naab 2005; Cox et al. 2006; Burkert et al. 2007). The mass ratio and the actual gas content have a significant influence on the final stellar orbit structure and internal kinematics of the merger remnants (Barnes \& Hernquist 1996; Jesseit et al. 2005, 2007; Thomas et al. 2007). Apparently, binary mergers of disk galaxies with varying mass ratios are one reasonable mechanism for the formation of intermediateand low-mass ellipticals, whereas more massive slowly rotating ellipticals are probably formed in multiple mergers at high redshifts (Naab et al. 2007; Burkert et al. 2007). A potential assembly mechanism for massive boxy and slowly-rotating elliptical galaxies at low redshift is merging of gas-poor early-type galaxies (dry mergers) (Naab et al. 2006b). There is now mounting observational evidence for dry galaxy mergers both in galaxy clusters van Dokkum et al. 1999; Tran et al. 2005) and in the field (van Dokkum 2005; Bell et al. 2006). Simulations of gas-free mergers have been shown to preserve the fundamental plane of elliptical galaxies (Nipoti et al. 2003; Bovlan-Kolchin et al. 2005). Furthermore Robertson et al. (2006b) showed that E-E mergers including $\mathrm{BH}$ feedback physics do not strongly alter the original $M_{\mathrm{BH}}-\sigma$ relation, although they might be the source for some of the observed scatter in the relation. Mixed merging, in which a gas-poor early-type galaxy merges with a gas-rich spiral disk galaxy might also form elliptical galaxies with distinct properties. They were shown by Khochfar \& Burkert (2003, 2005); Naab et al. (2006b); Hopkins et al. (2008a) to dominate the merger budget at intermediate masses between the low-mass ellipticals Sp-Sp mergers and the highest mass E-E mergers. Thus it is of utmost importance to study whether the current $\mathrm{BH}$ feedback model that reproduces the $M_{\mathrm{BH}}-\sigma$ and $M_{\mathrm{BH}}-M_{*}$ relations for equal-mass disk mergers, is also able to reproduce the observed relations in galaxy mergers with varying mass ratios and progenitor morphologies.

In this paper we present simulations of unequal-mass disk mergers, mixed E-Sp mergers and E-E mergers, including $\mathrm{BH}$ feedback. Our aim is to study the observed local $M_{\mathrm{BH}}-\sigma$ and $M_{\mathrm{BH}}-M_{*}$ relations and see whether they hold for our merger sample with varying mass ratios and different progenitor morphologies. We also study in detail the effect of varying the merger mass ratio on the peak $\mathrm{BH}$ accretion rate and final $\mathrm{BH}$ mass. In addition, we analyse the termination of star formation by $\mathrm{BH}$ feedback in unequal-mass disk mergers, mixed E-Sp mergers and dry E-E mergers compared to equal-mass disk mergers (Springel et al. 2005a). Furthermore, we discuss in detail the modeling of black hole mergers in the Springel et al. (2005b) feedback prescription. The merging of black holes is not resolved in the numerical simu- 
lations and therefore an effective model for $\mathrm{BH}$ merging needs to be adopted. We show that the assumption of rapid merging of the black holes in equal- and unequalmass galaxy mergers is critical for reproducing the final $\mathrm{BH}$ mass that fulfills the observed relations using the selfregulated Springel et al. (2005b) BH feedback model.

This paper is structured as follows. In $\$ 2$ we describe the simulation code and discuss the $\mathrm{BH}$ feedback and merger prescriptions. The galaxy model and merger setups, together with the adopted parameter settings, are discussed in $\$ 3$. Here we also simulate a set of isolated disk galaxies and perform a detailed analysis of the effect of the merger prescription and resolution on the growth and final mass of the BHs. In 4 we present the results and implications of our Sp-Sp, mixed E-Sp and dry E-E simulation sets to the origin of the $M_{\mathrm{BH}}-\sigma$, the $M_{\mathrm{BH}}-M_{*}$ and the $M_{\mathrm{BH}}-M_{*} \sigma^{2}$ relations. In addition we perform here a detailed study of $\mathrm{BH}$ and star formation activity as a function of merger mass ratio. Finally, we summarize and discuss our findings in $\$ 5$.

\section{METHODOLOGY}

\subsection{Numerical code}

We perform the simulations using the parallel TreeSPH-code GADGET-2 (Springel 2005). The code follows the gas dynamics using the Lagrangian Smoothed Particle Hydrodynamics (SPH) (e.g. Monaghan 1992) technique formulated in such a way that energy and entropy is manifestly conserved (Springel \& Hernquist 2002). Following Katz et al. (1996) the radiative cooling for a primordial mixture of hydrogen and helium together with a spatially uniform time-independent local UV background (Haardt \& Madau 1996) is included.

We include star formation and the associated supernova feedback, but exclude supernova-driven galactic winds, following the sub-resolution multiphase model developed by Springel \& Hernquist (2003). In this model the ISM is treated as a two-phase medium in which cold clouds are embedded in a tenous hot gas at pressure equilibrium. Stars form from the cold clouds in regions were $\rho>\rho_{\text {th }}$ with the shortlived stars supplying an energy of $10^{51} \mathrm{ergs}$ to the surrounding gas by supernovae. The threshold density, $\rho_{\mathrm{th}}$, is determined self-consistently in the model by requiring that the equation-of-state (EOS) is continuous at the onset of star formation.

We have implemented all the basic aspects of the Springel et al. (2005b) effective BH feedback model into our version of GADGET-2. We summarize the main aspects of the BH feedback model in Appendix $\mathrm{A}$ In Appendix B we perform a direct comparison of our feedback implementation with the original model and find that the two models are in very good agreement.

\subsection{Modeling black hole mergers}

In the final stages of a galaxy merger the dark matter halos of the galaxies merge and in the center a single stellar system is formed from the coalescence of the two stellar components. During this process a substantial fraction of the gaseous component is funneled to the center where it can drive strong starbursts and AGN activity. Presumably during this process the two central supermassive BHs form a binary, that hardens and eventually leads to a coalescence of the BHs. However, it is not clear how long this process would last and what the dominant mechanism for the hardening of the binary would be. Possible mechanisms include hardening of the BH binary through stellar-dynamical (Makino \& Funato 2004; Merritt \& Milosavljević 2005) and hydrodynamical (Escala et al. 2004) processes. At the final stages of the $\mathrm{BH}$ merger $(d \lesssim 0.01 \mathrm{pc})$ gravitational radiation will dominate angular momentum and energy losses and cause the two BHs to coalesce (e.g. Flanagan \& Hughes 1998). However for unequal-mass BH pairs, gravitational wave emission also removes net linear momentum and can impart a recoil velocity to the center of mass of the system. This recoil velocity is typically of the order of $v_{\mathrm{CM}} \sim 100-400 \mathrm{kms}^{-1}$ and may play a role in removing relatively low-mass supermassive BHs from dwarf galaxies at moderately to high redshifts (Madau \& Quataert 2004; Merritt et al. 2004).

In the simulations presented in this paper we lack the required resolution to follow the hardening and eventual merging of the BHs. Using current numerical techniques it is not possible to accurately resolve the dynamical evolution of a galactic BH pair on the relevant scales, although alternative techniques such as the particlesplitting method (Mayer et al. 2007) and adaptive mesh refinement simulations (Levine et al. 2008) might allow one to follow the $\mathrm{BH}$ dynamics to somewhat smaller separations compared to traditional methods. Instead, as was the case for the BH accretion, we use an effective model that ensures rapid merging of the $\mathrm{BHs}$ once they come sufficiently close to each other. There is circumstantial evidence suggesting that the merging of $\mathrm{BHs}$ is efficient. If this was not the case, it should be common for a third black hole to be brought in by merger, in which case the $\mathrm{BH}$ binary and third $\mathrm{BH}$ could lead to sling-shot ejection of all three BHs (Saslaw et al. 1974). This process would seriously hamper the growth of supermassive black holes. Thus the very existence of supermassive black holes together with the relatively low number of known supermassive BH binaries (Sillanpää et al. 1988; Merritt \& Milosavliević 2005) can be taken as an indication that $\mathrm{BH}$ binaries typically merge efficiently. Upcoming gravitational wave experiments, such as LISA will bring more clarity to the duration and true number of BH mergers in the local Universe (Hughes 2002).

In this study we adopt the criterion of Springel et al. (2005b) for BH merging, which allows for rapid BH merging. Two $\mathrm{BHs}$ are assumed to merge instantly if they come within the smoothing length of each other and if their relative velocity at this time is below the local sound speed, as determined from the nearest neighbors within the SPH kernel. The momentum is conserved when gas is absorbed by the $\mathrm{BH}$ sink particle, thus an accreting $\mathrm{BH}$ moving in a gas-rich environment will experience a friction force that reduces the relative motion of the $\mathrm{BH}$ with respect to the surrounding gas. However, we found that for unequal-mass mergers this description was not always adequate in ensuring rapid merging of the black holes. Typically the black holes had too large relative velocities to allow for merging and thus an alternative method had to be employed to ensure the merging of the BHs. After discussions with Volker Springel (Springel, private communication) we decided to adopt a model, in which the $\mathrm{BH}$ is repositioned at every time step to the minimum of the local potential. This minimum is evaluated by find- 
ing the gas particle within the SPH smoothing length of the $\mathrm{BH}$ which has the minimum gravitational potential. The $\mathrm{BH}$ is then repositioned to the location of this minimum at every timestep. This method effectively glues the $\mathrm{BH}$ to the center of the galaxy. Using this description the $\mathrm{BH}$ will remain in the merging galaxies as long as the galaxy core structure is intact. After disruption the two $\mathrm{BHs}$ will rapidly slide to the center of the parent galaxy and merge. Although this method is crude it should provide an adequate representation of a model, where one assumes that $\mathrm{BH}$ merging is very efficient once the two $\mathrm{BH}$ s reside in the same parent galaxy.

\section{SIMULATIONS}

\subsection{Galaxy models}

Our progenitor galaxies are setup using the method described by Springel et al. (2005b). The virial mass and radius of each model is determined by the virial velocity $v_{\text {vir }}$ of the halo assuming a virial overdensity of $\Delta_{\text {vir }}=$ 200 using the following relations

$$
\begin{aligned}
M_{\mathrm{vir}} & =\frac{v_{\mathrm{vir}}^{3}}{10 G H_{0}}, \\
r_{\mathrm{vir}} & =\frac{v_{\mathrm{vir}}}{10 G H_{0}},
\end{aligned}
$$

where $H_{0}=71 \mathrm{kms}^{-1} \mathrm{Mpc}^{-1}$ is the present day Hubble parameter. Thus $v_{\text {vir }}$ sets the mass of the model. The virial radius together with the concentration parameter $c=r_{\text {vir }} / r_{\mathrm{S}}$ for a NFW halo (Navarro et al. 1997) is then used to construct Hernquist (1990) profile dark matter halos using the conversion detailed in Springel et al. $(2005 \mathrm{~b})$. For all galaxy models we assume a concentration of $c=9$, which is a typical value for a halo at $z=0$ (Bullock et al. 2001). All of our model galaxies thus correspond to local $z=0$ disk galaxies and unlike Robertson et al. (2006c) we do not attempt a redshiftdependent scaling of our initial conditions.

The dark matter halos are then populated with exponential disks with a constant mass fraction of $m_{d}=0.041$ of the total virial mass resulting in a total disk mass of $M_{d}=m_{d} M_{\text {vir }}$ with a fractional gas content of $f_{\text {gas }}$ and the rest being stars. The disk scale length $r_{d}$ is determined using the Mo et al. (1998) formalism under the assumption that the fractional disk angular momentum $j_{d}$ equals the disk mass fraction $m_{d}$ for a constant halo spin of $\lambda=0.033$ for all models. This assumption of $J_{d}=j_{d} J_{\text {halo }}\left(j_{d}=m_{d}\right)$ corresponds to the conservation of the specific angular momentum of the material that forms the disk. The value for the spin parameter is close to the mean value found in numerical simulations (e.g. Vitvitska et al. 2002). The vertical scaleheight $z_{0}$ of the stellar disk is radially constant and set to $0.2 r_{d}$ and the radial velocity dispersion is set to equal the vertical velocity dispersion. The vertical scale height of the gaseous disk is set via an integral constraint on the surface mass density, a self-consistently determined galaxy potential and the effective equation of state (EOS) of the multiphase interstellar medium model (McKee \& Ostriker 1977; Efstathiou 2000; Johansson \& Efstathiou 2006). For the EOS we adopt the strongly pressurized multiphase model of the ISM, $q_{\mathrm{EOS}}=1$ (Springel \& Hernquist 2003) that allows the construction of stable galaxy mod- els even with very large gaseous fractions (Springel et al. 2005b).

All our galaxy models include a Hernquist (1990) stellar bulge component with constant total mass fraction of $m_{b}=0.01367\left(M_{b}=m_{b} M_{\mathrm{vir}}\right)$, which is a third of the disk mass fraction. The bulge scale length $b$ is also set to $0.2 r_{d}$. Finally we insert a seed black hole with mass $M_{\mathrm{BH}}=10^{5} M_{\odot}$ at rest in the center of each galaxy model.

\subsection{Simulation parameters}

At our standard numerical resolution we set the primary galaxy models up with 20,000 gas and stellar disk particles, 10,000 bulge particles and 30,000 dark matter particles. For the secondary galaxies in 3:1 mergers we use a third of the particle resolution of the primary galaxies, thus resulting in 6,667 gas and stellar disk particles, 3,333 bulge particles and 10,000 dark matter particles. We set the gravitational softening length of gas, newly formed stars and the black hole particles to $\epsilon=0.1 h^{-1} \mathrm{kpc}$ and scale the softening lengths of the disk, bulge and more massive dark matter particles with the square root of the particle mass resulting in $\epsilon=0.2 h^{-1} \mathrm{kpc}$ for the bulge and disk particles and $\epsilon=0.8 h^{-1} \mathrm{kpc}$ for the dark matter particles. This ensures that the maximum gravitational force exerted from a particle is independent of its mass (Dehnen 2001). Naab et al. (2006a) also showed that small variations in the respective softening lengths have only a minor effect on the central gas content, with typically only a $10 \%$ increase in the central gas content when equal softening lengths and mass resolution were employed for all collisionless particles. The SPH properties of the gas and $\mathrm{BH}$ particle are averaged over the usual GADGET-2 spline SPH kernel using $\sim 64$ SPH gas particles. Furthermore we require that the minimum SPH smoothing length is equal to the gravitational softening length. This choice safeguards against artificial stabilization of small clumps at low resolution (Bate \& Burkert 1997). In order to resolve the flow of gas around the black hole accurately we ran all simulations with high force accuracy of $\alpha_{\text {force }}=0.005$ and time integration accuracy of $\eta_{\text {acc }}=0.02$ (see Springel 2005 for details).

The parameters governing the multi-phase feedback model are set as follows: The star formation timescale $t_{*}^{0}=8.4 \mathrm{Gyr}$, the cloud evaporation parameter $A_{0}=$ 4000 and the supernova 'temperature' $T_{\mathrm{SN}}=4.0 \times 10^{8} \mathrm{~K}$ that reflects the heating rate from a population of supernovae for a given IMF. Thus, we here again follow Springel et al. (2005b) who showed that these choices result in a star formation rate of $\sim 1 M_{\odot} \mathrm{yr}^{-1}$ for a Milky Way-like galaxy. However, we note that these choices yield a comparatively low star formation rate that lies slightly below the observed Kennicutt relation (Kennicutt 1998).

We set the dimensionless efficiency parameter of accretion in Eq. A1 to $\alpha=100$, which is considerably higher than the theoretically expected value of $\alpha \sim 1$. This discrepancy is essentially due to limits imposed by the numerical resolution and the effective subresolution model for star formation. The estimated values for the gas density and thermal sound speed are large-scale averages of the gas at the location of the $\mathrm{BH}$ resulting in artifically low densities and high sound speeds compared to the case 


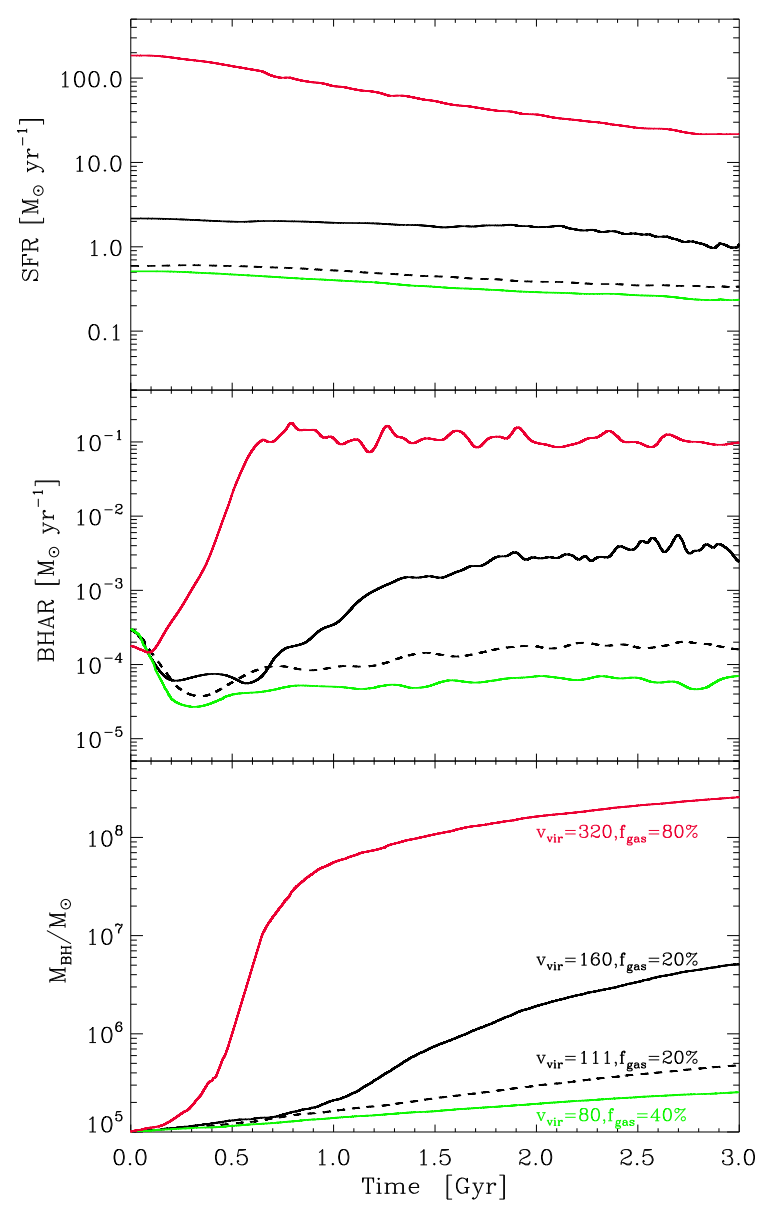

FIG. 1. - The star formation rate (top), the black hole accretion rate (middle) and the evolution of the black hole mass (bottom) as a function of time for four isolated galaxy models with initial gas mass fractions of $20 \%$ (black), $40 \%$ (green) and $80 \%$ (red) and virial velocities as indicated in the Figure.

where the multi-phase structure of the ISM could be spatially resolved on the scale of the Bondi radius. Thus the larger value for $\alpha$ can essentially be seen as an empirical correction factor that translates from the resolvable low mean density to the time-averaged small-scale density at the location of the BH. A similar conclusion was reached by Khalatvan et al. (2008), who set $\alpha=300$ in their simulations. The exact choice of $\alpha$ within a factor of a few is not critical as long as $\alpha$ is large enough to allow a black hole in a gas-rich environment to reach the Eddington regime, (again see Springel et al. 2005b for details). As detailed in $\mathrm{A}$ we set the radiative efficiency to $\epsilon_{\mathrm{r}}=0.1$ and the thermal coupling constant of the black hole feedback energy to $\epsilon_{f}=0.05$.

All simulations presented in this paper were evolved for a total of $t=3 \mathrm{Gyr}$ using the local Altix $3700 \mathrm{Bx} 2$ machine hosted at the University Observatory in Munich.

\subsection{Isolated disks with $\mathrm{BHs}$}

We begin by running a representative sample of our disk galaxy models in isolation in order to study the stability of the constructed galaxy models. The models are run at our standard numerical resolution. In Fig. 1] we show the resulting star formation rates, $\mathrm{BH}$ accretion rates and $\mathrm{BH}$ mass growth for four models with $20 \%$ (black), 40\% (green), 80\% (red) initial gas mass frac-
TABLE 1

Isolated GALAXies AND THE $M_{\mathrm{BH}}-\sigma$ AND $M_{\mathrm{BH}}-M_{*}$ RELATIONS

\begin{tabular}{ccccccc}
\hline \hline$v_{\text {vir }}{ }^{a}$ & $f_{\text {gas }}$ & $M_{\mathrm{BH}^{b}}$ & $\sigma_{\text {bulge }^{c}}$ & $M_{\text {bulge }^{d}}$ & $\frac{M_{\mathrm{BH}}}{M_{\mathrm{BH}, \sigma}}{ }^{e}$ & $\frac{M_{\mathrm{BH}}}{M_{\mathrm{BH}, \mathrm{M}_{*}}}$ \\
\hline 80 & 0.4 & 5.12 & 57.0 & 0.23 & 0.59 & 0.22 \\
111 & 0.2 & 6.50 & 78.3 & 0.61 & 0.21 & 0.09 \\
160 & 0.2 & 56.0 & 120.4 & 1.83 & 0.32 & 0.24 \\
320 & 0.8 & 2640 & 252.9 & 14.7 & 0.76 & 1.08 \\
\hline
\end{tabular}

${ }^{a}$ Virial velocity in $\left[\mathrm{kms}^{-1}\right]$

${ }^{b}$ Final mass of the $\mathrm{BH}$ in $10^{5} \mathrm{M}_{\odot}$

${ }^{c}$ Final bulge stellar velocity dispersion in $\mathrm{km} / \mathrm{s}$

${ }^{d}$ Final bulge mass in $10^{10} M_{\odot}$

${ }^{e}$ The ratio of the final $\mathrm{BH}$ mass to the $\mathrm{BH}$ mass expected from Eq. 3 if the final $\mathrm{BH}$ would lie on the $M_{\mathrm{BH}}-\sigma$ relation.

${ }^{f}$ The ratio of the final $\mathrm{BH}$ mass to the $\mathrm{BH}$ mass expected from Eq. 4 if the final $\mathrm{BH}$ would lie on the $M_{\mathrm{BH}}-M_{*}$ relation

tions. All galaxies show stable evolution in their star formation and $\mathrm{BH}$ accretion rates, reproducing the isolated disk results for a stiff EOS with $q_{\mathrm{EOS}}=1$ presented by Springel et al. (2005b).

All models start with a seed black hole with mass $M_{\mathrm{BH}}=10^{5} M_{\odot}$ at rest in the center, which grows due to gas accretion during the simulation as is shown in the bottom panel of Fig. 1. After completing the isolated disk simulations we calculate the final black hole mass of the galaxies together with the mass-weighted line-of-sight stellar velocity dispersion $\sigma_{\text {bulge }}$ measured from the bulge stellar particles using 50 randomly projected realizations of the galaxy models. Using the extracted velocity dispersions we then calculate the expected $\mathrm{BH}$ masses of the galaxies and check if they lie on the observed $M_{\mathrm{BH}}-\sigma$ relation,

$$
\log \left(M_{\mathrm{BH}} / M_{\odot}\right)=a+b \log \left(\sigma / \sigma_{0}\right),
$$

where the relation is defined relative to $\sigma_{0}=200 \mathrm{kms}^{-1}$. The observed values as determined by Tremaine et al. (2002) are $a=8.13 \pm 0.06$ for the normalization coefficient and $b=4.02 \pm 0.32$ for the slope. We also evaluate the total bulge stellar mass $M_{\text {bulge }}$ by adding up the mass of all the stellar bulge particles. By definition this mass is not changing throughout the simulation, as the newly formed stars form from gas in the disk and we do not include their potential contribution to the final stellar bulge mass. We then compare the final $\mathrm{BH}$ masses with the expected $\mathrm{BH}$ masses if the galaxies would lie on the observed $M_{\mathrm{BH}}-M_{*}$ relation, where we now use $M_{*}$ to denote the total bulge mass.

$$
\log \left(M_{\mathrm{BH}} / M_{\odot}\right)=c+d \log \left(M_{*} / 10^{11} M_{\odot}\right) .
$$

The best fit observed values as determined by Häring \& Rix (2004) are $c=8.20 \pm 0.10$ for the normalization coefficient and $d=1.12 \pm 0.06$ for the slope. The final BH masses, stellar velocity dispersions, final stellar masses and the ratios between the final $\mathrm{BH}$ mass and the $\mathrm{BH}$ mass derived from Eqs. 3 and 4 assuming that the galaxies lie on the $M_{\mathrm{BH}}-\sigma$ and $M_{\mathrm{BH}}-M_{*}$ relations respectively are tabulated in Table 1.

The initial seed mass of $M_{\mathrm{BH}}=10^{5} M_{\odot}$ corresponds to a velocity dispersion of $\sigma=33 \mathrm{kms}^{-1}$ (Eq. 3), which would be the corresponding velocity dispersion of a galaxy with $v_{\text {vir }} \sim 50 \mathrm{kms}^{-1}$. Thus the BHs of the galaxies are not initially on the $M_{\mathrm{BH}}-\sigma$ relation, but evolve towards this relation during the simulation. The 
bulge velocity dispersions of the galaxies increase only slightly on the $\sim 10 \%$ level during the simulations. The governing factor determining the evolution of the $\mathrm{BHs}$ for the isolated disk galaxies is the depth of the potential well and the gas mass fraction. This can be seen in the ratio of the final $\mathrm{BH}$ mass to the expected $\mathrm{BH}$ mass from Eq. 3 tabulated in Table1. The lower the gas mass fraction the lower the final $\mathrm{BH}$ masses are with respect to the expected $M_{\mathrm{BH}}-\sigma$ relation. Models with lower gas mass fractions would require additional gas inflow to the central regions in order to ensure sufficient $\mathrm{BH}$ growth. Such additional gas inflow could be triggered by galaxy mergers. The same initial $\mathrm{BH}$ seed mass of $M_{\mathrm{BH}}=10^{5} M_{\odot}$ would require initially a total bulge mass of $M_{*}=1.4 \cdot 10^{8} M_{\odot}$. Again this would only be valid for the very lowest mass initial galaxy models and thus generally the galaxies do not initially lie on the $M_{\mathrm{BH}}-M_{*}$ relation. Typically the simulations of the isolated galaxies result in $\mathrm{BH}$ masses that are too low for their given stellar bulge masses. Again an additional central gas flow, potentially induced by a galaxy merger is required for the central BHs to reach the masses expected by their corresponding stellar bulge masses.

Interestingly the simulation of the most massive and gas-rich galaxy (the $f_{\text {gas }}=80 \%, v_{\text {vir }} \sim 320 \mathrm{kms}^{-1}$ model in Fig. 1 and Table 1) results in a final $\mathrm{BH}$ mass that is relatively close to the $\mathrm{BH}$ mass expected from the $M_{\mathrm{BH}}-\sigma$ relation and even superceeds the $\mathrm{BH}$ mass expected from the $M_{\mathrm{BH}}-M_{*}$ relation. However, some of this BH mass growth may be artificially high, due to the fact that the current $\mathrm{BH}$ feedback model does not restrict the size of the $\mathrm{BH}$ accretion radius. Thus the accretion radius of a a massive $\mathrm{BH}$ in a gas-rich region may grow to unphysically large sizes and accrete material that would physically not end up in the $\mathrm{BH}$ because of its high angular momentum and large distance from the $\mathrm{BH}$. This is typically not a problem in merger simulations, where most of the $\mathrm{BH}$ mass grows by accretion of nearby high-density gas.

\subsection{The effect of resolution on the $M_{\mathrm{BH}}-\sigma$ relation}

We then proceed with a detailed analysis of the effect of the $\mathrm{BH}$ merger prescription and resolution on the final mass of BHs and the corresponding stellar velocity dispersions in 3:1 and 1:1 merger remnants. We simulate 3:1 mergers with a quarter (tiny), half (low), double (medium) and four times (high) the number of particles compared to our standard numerical resolution (\$3.2). In addition we compute a control sample of 1:1 mergers at the standard, low and tiny resolutions. The five $3: 1$ and three 1:1 initial conditions are simulated using both the standard $\mathrm{BH}$ merging prescription and the $\mathrm{BH}$ repositioning method described in 22.2 resulting in a total of 16 simulations (see Table 2 for details). The gravitational softening lengths for all the particles including the $\mathrm{BH}$ are scaled with the inverse cube root of the number of particles, with larger numerical resolution resulting in smaller gravitational softening lengths. In addition we run a 3:1 and a 1:1 merger with a lower accretion efficiency of $\alpha=1$ at our standard resolution using the $\mathrm{BH}$ repositioning method.

All galaxy models have a $20 \%$ initial gas fraction, with $v_{\mathrm{vir}, \mathrm{p}}=160 \mathrm{kms}^{-1}, v_{\mathrm{vir}, \mathrm{s}}=111 \mathrm{kms}^{-1}$ and are setup with the parameters described in 3.1 ( $\mathrm{p}$ and $\mathrm{s}$ denote the

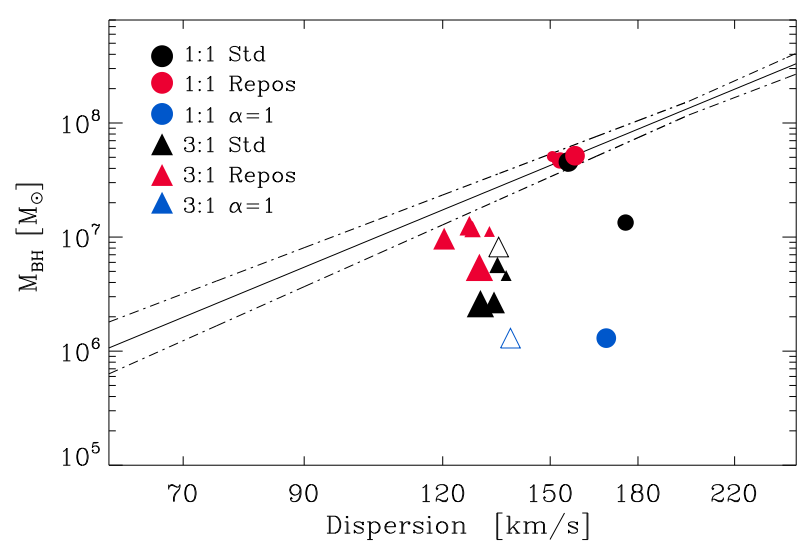

FIG. 2.- The $M_{\mathrm{BH}}-\sigma$ relation as a function of resolution and merger prescription, with larger/smaller symbols indicating higher/lower resolution, respectively (Table 2). Triangles show 3:1 and circles 1:1 merger remnants. Black symbols employ the standard merger prescription and the red symbols use the BH repositioning method. The blue symbols also employ the $\mathrm{BH}$ repositioning method but with a reduced accretion efficiency of $\alpha=1$. The solid/open symbols indicate cases where the BHs did/did not merge. (Table 2). The lines show the observed relation with errors by Tremaine et al. (2002).

primary and secondary galaxies, respectively). In order to isolate the effects of resolution and merging prescription all 3:1 and 1:1 mergers are run with identical merging geometries and initial disk orientations. For the orbit and initial orientation we adopt geometry G13 for both the 3:1 mergers and 1:1 mergers from Naab \& Burkert (2003). The G13 geometry corresponds to the inclinations $i_{p}=-109, i_{s}=180$ and the arguments of the pericenter $\omega_{p}=60, \omega_{s}=0$ for the primary and secondary galaxies, respectively. The galaxies approach each other on a parabolic orbit where the initial separation of the progenitors is $R_{\text {init }}=0.5\left(r_{\mathrm{vir}, \mathrm{p}}+r_{\mathrm{vir}, \mathrm{s}}\right)$ and the pericentric distance is $r_{\text {peri }}=r_{\mathrm{d}, \mathrm{p}}+r_{\mathrm{d}, \mathrm{s}}$, where $r_{\mathrm{vir}, \mathrm{p}}, r_{\mathrm{d}, \mathrm{p}}$ and $r_{\mathrm{vir}, \mathrm{s}}, r_{\mathrm{d}, \mathrm{s}}$ are the virial and disk scale radii for the primary and secondary galaxies, respectively. We note that radial parabolic or near-parabolic orbits are generally motivated by statistics from N-body simulations (Khochfar \& Burkert 2006).

After completing the merger simulations we calculate the final black hole mass together with mass-weighted line-of-sight stellar velocity dispersion $\sigma$ measured from all stellar particles within the projected half-mass radius $r_{\mathrm{e}}$ using 50 randomly projected realizations of the merger remnant. This determination of $\sigma$ is similar to the method used by observers who measure the luminosityweighted velocity dispersion within an effective radius (Gebhardt et al. 2000). The simulated final BH masses and stellar velocity dispersions together with the time of the BH merger are tabulated in Table 2 .

For the 3:1 mergers, the merging prescription has a strong effect on the final BH mass of the merger remnant, with the standard prescription producing $\mathrm{BH}$ masses that are a factor of 2-3 lower than the corresponding $\mathrm{BH}$ masses using the repositioning method. The final stellar velocity dispersion on the other hand converges with increasing resolution, with the repositioning method resulting in marginally lower velocity dispersions. The 3:1 mergers with the standard $\mathrm{BH}$ merger prescription result in $\mathrm{BH}$ masses that are too low for their corresponding 
Equal- and unequal-mass mergers with black holes

TABLE 2

3:1 AND 1:1 BINARY BH MERGER RESOLUTION AND MERGER PRESCRIPTION STUDY

\begin{tabular}{|c|c|c|c|c|c|c|c|c|c|c|c|}
\hline Resolution $^{a}$ & Mass ratio & $\epsilon_{\mathrm{gas}}{ }^{b c}$ & $\epsilon_{\text {disk }}{ }^{d}$ & $\epsilon_{\mathrm{DM}}$ & $N_{\text {gas }}{ }^{e}$ & $N_{\text {bulge }}$ & $N_{\mathrm{DM}}$ & $m_{\text {gas }}{ }^{f}$ & $M_{\mathrm{BH}, \mathrm{fin}^{g}}$ & $\sigma_{\text {fin }}^{h}$ & $t_{\text {merg }}$ \\
\hline Tiny Std & $3: 1$ & 0.16 & 0.32 & 1.28 & 5000 & 2500 & 7500 & 31 & 46.0 & 136.9 & 2.02 \\
\hline Tiny Repos & $3: 1$ & 0.16 & 0.32 & 1.28 & 5000 & 2500 & 7500 & 31 & 118 & 132.2 & 2.81 \\
\hline Low Std & $3: 1$ & 0.125 & 0.25 & 1.00 & 10000 & 5000 & 15000 & 15.5 & 57.0 & 134.4 & 2.00 \\
\hline Low Repos & $3: 1$ & 0.125 & 0.25 & 1.00 & 10000 & 5000 & 15000 & 15.5 & 118 & 127.7 & 2.50 \\
\hline Standard Std & $3: 1$ & 0.1 & 0.2 & 0.8 & 20000 & 10000 & 30000 & 7.75 & 81.7 & 134.7 & - \\
\hline Standard Repos & $3: 1$ & 0.1 & 0.2 & 0.8 & 20000 & 10000 & 30000 & 7.75 & 126 & 126.8 & 1.82 \\
\hline Medium Std & $3: 1$ & 0.08 & 0.16 & 0.64 & 40000 & 20000 & 60000 & 3.87 & 26.7 & 133.5 & 1.84 \\
\hline Medium Repos & $3: 1$ & 0.08 & 0.16 & 0.64 & 40000 & 20000 & 60000 & 3.87 & 96.9 & 120.3 & 1.72 \\
\hline High Std & $3: 1$ & 0.0625 & 0.125 & 0.5 & 80000 & 40000 & 120000 & 1.94 & 26.1 & 129.8 & 2.81 \\
\hline High Repos & $3: 1$ & 0.0625 & 0.125 & 0.5 & 80000 & 40000 & 120000 & 1.94 & 54.2 & 129.5 & 1.86 \\
\hline Tiny Std & $1: 1$ & 0.16 & 0.32 & 1.28 & 5000 & 2500 & 7500 & 31 & 420 & 155.4 & 2.21 \\
\hline Tiny Repos & $1: 1$ & 0.16 & 0.32 & 1.28 & 5000 & 2500 & 7500 & 31 & 508 & 150.7 & 1.57 \\
\hline Low Std & $1: 1$ & 0.125 & 0.25 & 1.00 & 10000 & 5000 & 15000 & 15.5 & 134 & 175.4 & 1.84 \\
\hline Low Repos & $1: 1$ & 0.125 & 0.25 & 1.00 & 10000 & 5000 & 15000 & 15.5 & 469 & 153.1 & 1.57 \\
\hline Standard Std & $1: 1$ & 0.1 & 0.2 & 0.8 & 20000 & 10000 & 30000 & 7.75 & 456 & 155.8 & 1.72 \\
\hline Standard Repos & $1: 1$ & 0.1 & 0.2 & 0.8 & 20000 & 10000 & 30000 & 7.75 & 516 & 157.9 & 1.50 \\
\hline
\end{tabular}

${ }^{a}$ The secondary has a third of the particles of the primary

${ }^{b}$ Gravitational softening lengths in $h^{-1} \mathrm{kpc}$

${ }^{c} \epsilon_{\text {gas }}=\epsilon_{\text {stars }}=\epsilon_{\mathrm{BH}}$

${ }^{d} \epsilon_{\mathrm{disk}}=\epsilon_{\mathrm{bulge}}$

${ }^{e} N_{\text {gas }}=N_{\text {disk }}$

$f_{\text {Mass of a gas particle in } 10^{5} M_{\odot}}$

${ }^{g}$ Final mass of the $\mathrm{BH}$ in $10^{5} M_{\odot}$

${ }^{h}$ Final stellar velocity dispersion within $r_{\text {eff }}$ in $\mathrm{km} / \mathrm{s}$

${ }^{i}$ Time of $\mathrm{BH}$ merger in Gyr

velocity dispersions as shown in Fig. 2, where the simulated 3:1 (triangles) and 1:1 (circles) results are overplotted on the observed $M_{\mathrm{BH}}-\sigma$ relation from Eq. 3 by Tremaine et al. (2002).

For the 1:1 mergers the effect of the merger prescription is less pronounced. With the exception of a single simulation at low resolution, all 1:1 mergers result in very similar BH masses and velocity dispersions. At the standard resolution the two prescriptions produce final $\mathrm{BH}$ masses and velocity dispersions that agree within $10 \%$ with each other and the observed relation. All the 1:1 mergers with repositioning and two out of three of the standard prescription mergers lie on the observed $M_{\mathrm{BH}}-\sigma$ relation as shown in Fig. 2,

Finally we show that reducing the accretion efficiency from our standard value of $\alpha=100$ to the physically motivated $\alpha=1$ produces $\mathrm{BH}$ masses that are far too low for their corresponding velocity dispersions (the blue points in Fig. 2). However, this is expected given the limitations imposed by the numerical resolution and subresolution modeling as was discussed in 3.2 Changing the accretion efficiency or even removing the $\mathrm{BH}$ altogether does not affect the final stellar velocity dispersion, which is primarily set by the depth of the potential well. Thus, this implies that the $\mathrm{BH}$ is not dynamically important for the galaxy as a whole. A similar problem would arise when comparing the simulated $\mathrm{BH}$ masses to the total stellar masses of the galaxies. Variations in numerical resolution and $\mathrm{BH}$ merging prescription affect only marginally the star formation rate and thus the $\mathrm{BH}$ masses in the standard prescription 3:1 merger remnants would be too low compared to their corresponding stellar masses.

The numerical resolution tests show that the central stellar velocity dispersion converge within $10 \%$ with increasing resolution, whereas the $\mathrm{BH}$ mass converges within $25 \%$, with the exception of the highest resolution simulation using the repositioning method. The highest resolution repositioning run produces a $\mathrm{BH}$ mass, which is lower by a factor of two compared to the other simulations. This is mainly due to the reduction of the gravitational softening of the $\mathrm{BH}$ particle. The accretion rate and mass growth of the $\mathrm{BH}$ is determined by a combination of the accretion efficiency ( $\alpha$ in Eq. A1) and the gravitational softening. Our chosen value of accretion efficiency was adopted for our standard numerical resolution, thus it is not surprising that smaller gravitational softening lengths produce slightly smaller BH masses.

In Appendix $\mathrm{C}$ we study in more detail the differences between the standard and repositioning merger prescription for both 3:1 and 1:1 binary galaxy mergers with BHs. We find that the repositioning merger prescription at the standard numerical resolution produces stable results and thus we adopt this prescription and numerical resolution for all remaining simulations presented in this paper.

\section{RESULTS}

\subsection{Unequal-mass 3:1 disk mergers with BHs}

Using the galaxy models described in 3.1 we set up five unequal-mass 3:1 mergers and five equal-mass 1:1 mergers with three different initial gas mass fractions of $f_{\text {gas }}=0.2,0.4,0.8$, resulting in a total of thirty simulations (the S1- and S2-series in Table 3). The progenitor galaxies are at the standard numerical resolution with the virial velocities of the models ranging from $v_{\text {vir,p }}=50-500 \mathrm{kms}^{-1}$ and $v_{\text {vir, }}=35-347 \mathrm{kms}^{-1}$ for the primary and secondary galaxies, respectively. The progenitors merge on prograde, nearly parabolic, in plane orbits (G0 orbit), with inclinations $i_{1}=i_{2}=0$ and arguments of the pericenter $\omega_{1}=\omega_{2}=0$. The initial separation and pericentric distances are set to the mean of the virial radius and twice the mean of the scale radius respectively, as detailed in 3.4 . In addition we simulate a set of three $3: 1$ and three 1:1 mergers with varying initial disk geometries and orbits. These simulations (the G1- 
TABLE 3

UNEQUAL- AND EQUAL-MASS MERGERS

\begin{tabular}{|c|c|c|c|c|c|c|c|c|c|}
\hline Models & $v_{\mathrm{vir}, \operatorname{prog} 1^{a}}$ & $v_{\text {vir,prog } 2}$ & Mass ratio & Orbit & $f_{\text {gas }}$ & $N_{\text {gas }, \text { tot }}{ }^{b}$ & $N_{\text {bulge, tot }}$ & $N_{\text {DM, tot }}$ & $N_{\mathrm{sim}}$ \\
\hline $\mathrm{S} 1-1: 1$ & $50,80,160,320,500$ & $50,80,160,320,500$ & $1: 1$ & G0 & $0.2,0.4,0.8$ & 40000 & 20000 & 60000 & 15 \\
\hline S2-3:1 & $50,80,160,320,500$ & $35,55,111,222,347$ & $3: 1$ & Go & $0.2,0.4,0.8$ & 26667 & 13333 & 40000 & 15 \\
\hline G1-1:1 & 160 & 160 & $1: 1$ & G7, G10, G13 & 0.2 & 40000 & 20000 & 60000 & 3 \\
\hline G2-3:1 & 160 & 111 & $3: 1$ & G4, G10, G14 & 0.2 & 26667 & 13333 & 40000 & 3 \\
\hline
\end{tabular}

${ }^{a}$ All virial velocities in $\mathrm{kms}^{-1}$

${ }^{b} N_{\text {gas }}=N_{\text {disk }}$

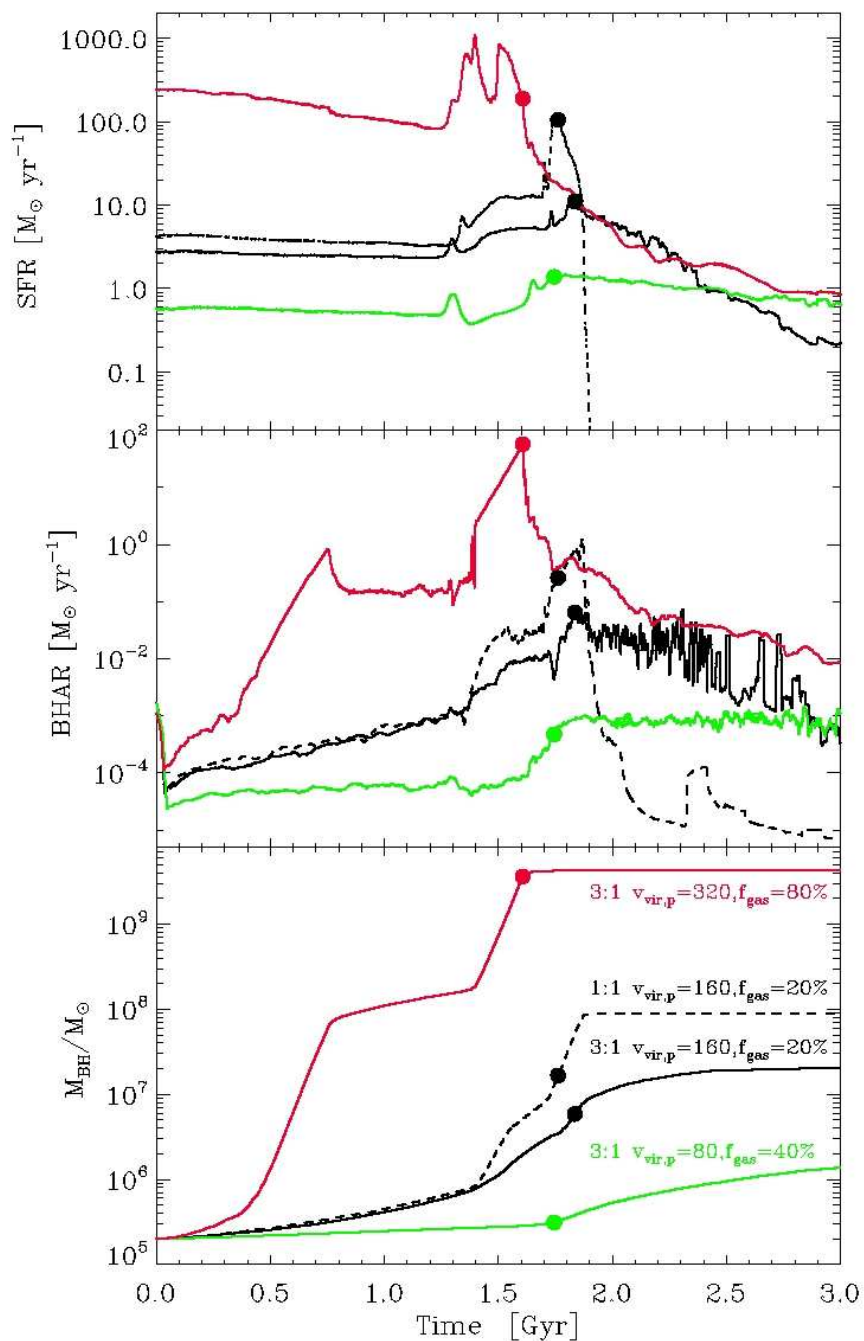

FIG. 3.- The total star formation rate (top), the total black hole accretion rate (middle) and the evolution of the total black hole mass (bottom) as a function of time for three 3:1 (solid lines) and one 1:1 (dashed line) merger with initial gas mass fractions of $20 \%$ (black), $40 \%$ (green) and $80 \%$ (red). The filled circles indicate the time of merging of the BHs.

and G2-series in Table 3) were all run with $f_{\text {gas }}=0.2$ and $v_{\text {vir,p }}=160 \mathrm{kms}^{-1}, v_{\text {vir,s }}=111 \mathrm{kms}^{-1}$ progenitors on the G7, G10, G13 orbits for the equal-mass mergers and the G4, G10, G14 orbits for the unequalmass mergers (see Naab \& Burkert 2003 for a definition of the orbital parameters). The orbits in the G-series were chosen to provide a good coverage of the expected anisotropy-ellipticity $(\delta-\epsilon)$ plane for merger remnants (see Burkert et al. 2007 for definitions of $\delta$ and $\epsilon$ ).

In Fig. 3 we plot the total star formation rates, $\mathrm{BH}$ accretion rates and the $\mathrm{BH}$ mass growth for three 3:1 merg-

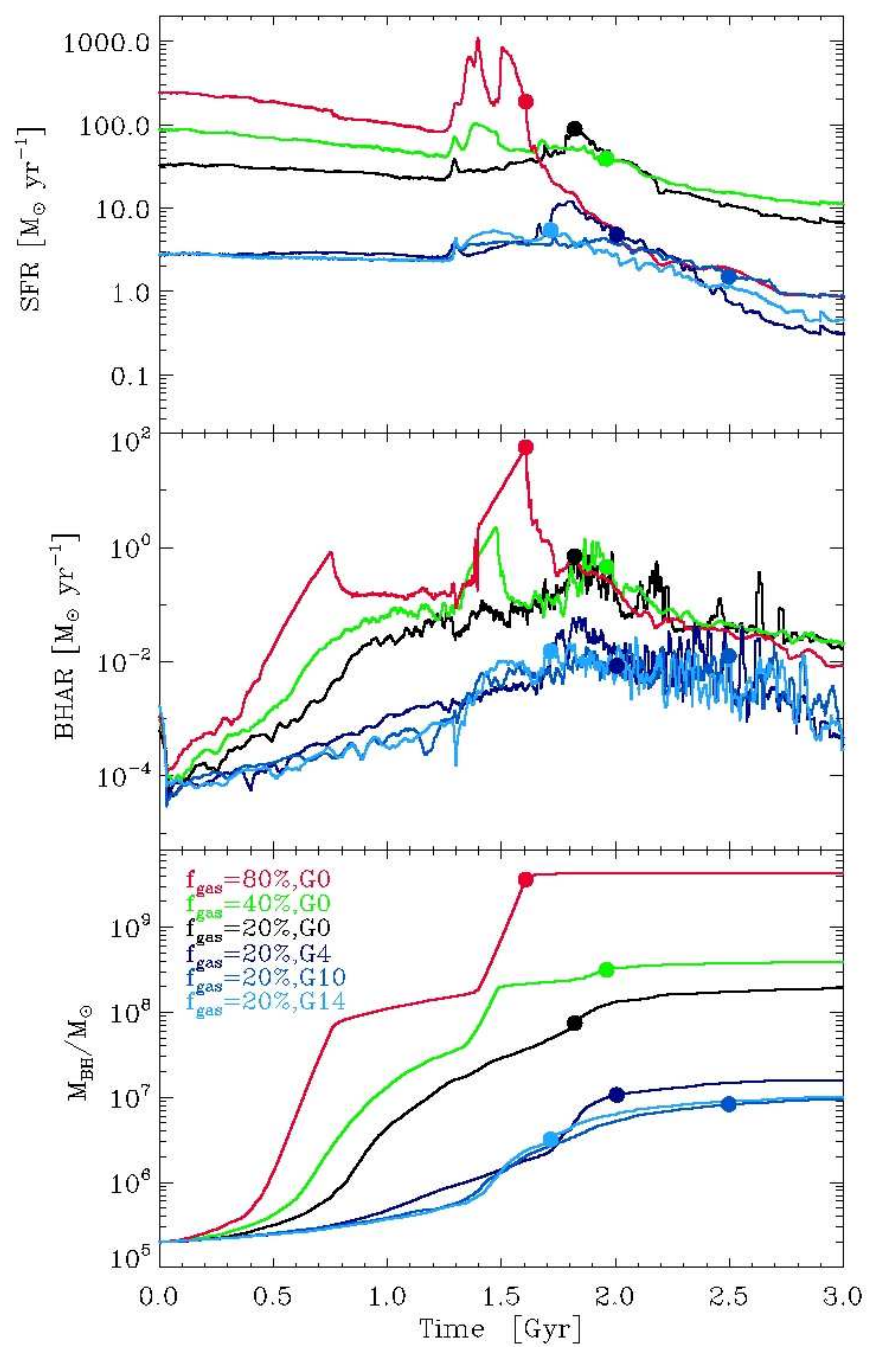

FIG. 4. - The total star formation rate (top), the total black hole accretion rate (middle) and the evolution of the total black hole mass (bottom) as a function of time for three 3:1 mergers with varying initial gas mass fractions (black, green, red lines) and three 3:1 mergers with varying orbital and initial geometries for a fixed gas fraction (blue lines).

ers (solid lines) with 20\% (black), 40\% (green), $80 \%$ (red) initial gas mass fractions together with one $20 \%$ gas fraction 1:1 merger (dashed lines). The star formation is very efficiently terminated by the $\mathrm{BH}$ feedback in 1:1 mergers, compared to a generally much shallower decline in star formation for 3:1 mergers. In the lowest mass 3:1 merger the $\mathrm{BH}$ feedback is actually unable to terminate the star formation and the galaxy is forming stars at a constant rate even after the $\mathrm{BH}$ merger. A similar trend is true for the $\mathrm{BH}$ accretion rates, with a much gentler decline in the accretion rate for galaxies undergoing 3:1 mergers 

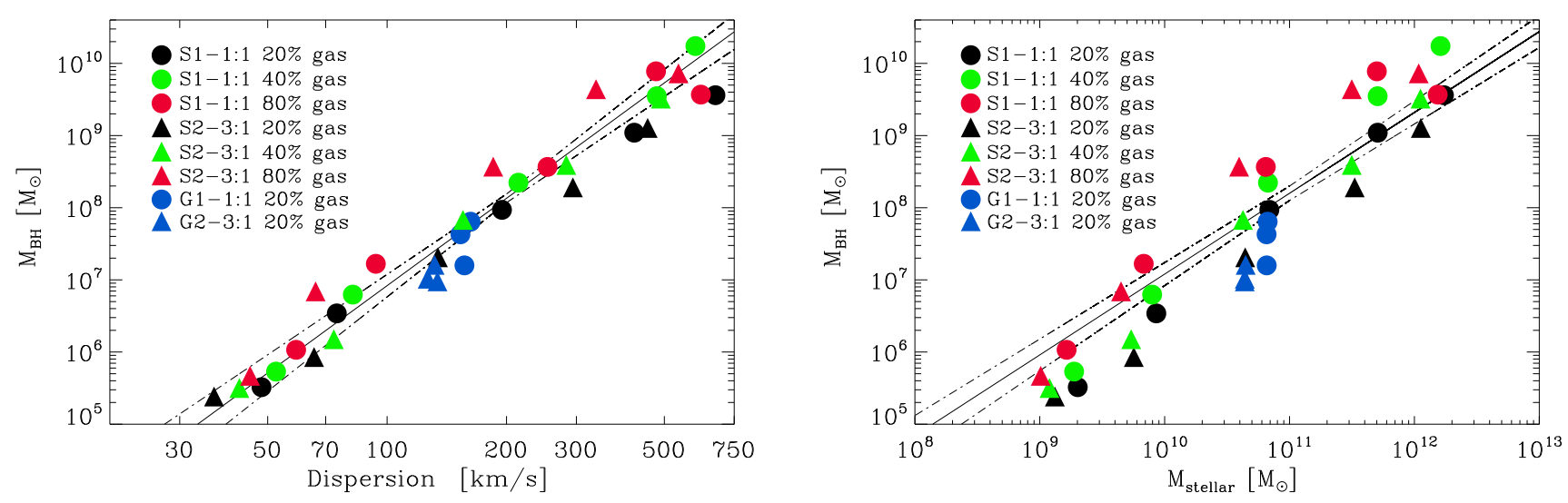

FIG. 5.- The $M_{\mathrm{BH}}-\sigma$ (left) and $M_{\mathrm{BH}}-M_{*}$ (right) relations for our complete sample of 3:1 (triangles) and 1:1 (circles) mergers. The black, green and red symbols show the effect of varying the initial gas mass fraction for our S1- (equal-mass) and S2-samples (unequal-mass). The blue symbols show the variation caused by the orbit and initial geometry for our G1- and G2-samples (see also Table 3). The lines show the observed relations with errors by Tremaine et al. (2002) (left panel) and Häring \& Rix (2004) (right panel), respectively.

TABLE 4

BEST FIT $M_{\mathrm{BH}}-\sigma$ RELATION FOR 3:1 AND 1:1 MERGERS

\begin{tabular}{ccccc}
\hline \hline Sample & $\mathrm{N}$ & $a$ & $b$ & $\Delta_{\log M_{\mathrm{BH}}}$ \\
\hline Tot sample & 36 & $8.07 \pm 0.06$ & $3.82 \pm 0.15$ & 0.29 \\
3:1 sample & 18 & $8.06 \pm 0.08$ & $3.78 \pm 0.18$ & 0.33 \\
1:1 sample & 18 & $8.05 \pm 0.07$ & $3.77 \pm 0.18$ & 0.26 \\
S1-S2 20\% gas sample & 10 & $7.85 \pm 0.04$ & $3.47 \pm 0.12$ & 0.13 \\
S1-S2 40\% gas sample & 10 & $8.13 \pm 0.05$ & $3.96 \pm 0.13$ & 0.14 \\
S1-S2 80\% gas sample & 10 & $8.35 \pm 0.10$ & $3.77 \pm 0.28$ & 0.29 \\
Observed sample ${ }^{a}$ & 31 & $8.13 \pm 0.06$ & $4.02 \pm 0.32$ & $0.25-0.3$ \\
\hline${ }^{a}$ From Tremaine et al. $(2002)$.
\end{tabular}

compared to 1:1 mergers. This evolution is mirrored in the growth of the $\mathrm{BH}$ masses, with the final $\mathrm{BH}$ mass being lower in 3:1 mergers typically by a factor of $2-5$, but with a relatively large scatter depending on the progenitor masses and initial gas fractions. Most of the mass growth of the BHs typically takes place shortly after the merging of the BHs as indicated by the filled circles in Fig. 3 ,

In Fig. 4 we show the results for three 3:1 mergers with varying initial gas mass fractions for a fixed orbit and initial disk geometry (black, green, red lines) and for three 3:1 mergers with varying initial orbits and orientations for a fixed gas mass fraction (blue lines). The initial gas fraction has a large effect on the height of the star formation and $\mathrm{BH}$ accretion peaks, with larger initial gas fractions producing higher values, as expected. This results also in relatively large differences in the final $\mathrm{BH}$ masses, with the $f_{\text {gas }}=0.8$ simulations producing final $\mathrm{BH}$ masses that are larger by an order of magnitude compared to the $f_{\text {gas }}=0.2$ runs. The variation of the orbit and initial geometry for a fixed gas mass fraction produces much smaller differences. The peaks of the star formation rates and $\mathrm{BH}$ accretion rates only vary within a factor of two with changing orbits and initial disk geometries. And although the time of the $\mathrm{BH}$ merging varies somewhat between different geometries the final $\mathrm{BH}$ mass is virtually unchanged. This was already shown to be the case for 1:1 mergers by Springel et al. (2005b) and Fig. 4 confirms the same conclusion for 3:1 mergers. Hence the final $\mathrm{BH}$ mass is sensitive to the initial gas mass fraction of the galaxies, but relatively insensitive to the initial orbit and orientation of the galaxies.
Figure 5 shows the $M_{\mathrm{BH}}-\sigma$ (left panel) and $M_{\mathrm{BH}}-$ $M_{*}$ (right panel) relations, extracted from our total sample of 36 simulated mergers, with the filled triangles and circles depicting 3:1 and 1:1 merger remnants, respectively, together with the observed relations by Tremaine et al. (2002) and Häring \& Rix (2004). We perform a Levenberg-Marquardt least-squares fit to the $M_{\mathrm{BH}}-\sigma$ relation as defined in Eq. 3. Table 4 lists the best fit values for the normalization coefficient $a$ and the slope $b$ together with their $1 \sigma$ uncertainties derived using the Bootstrap method. In addition we list the dispersion $\Delta_{\log M_{\mathrm{BH}}}$ about each best-fit relation. The fitting analysis was performed for our total merger sample, for the $3: 1$ and 1:1 samples separately and for the sample split as a function of the initial gas mass fraction. Our best fit normalization coefficient and slopes for the total sample are marginally lower compared to the observational values of Tremaine et al. (2002), but still well within the error limits. Furthermore the intrinsic $M_{\mathrm{BH}}-\sigma$ dispersion induced primarily by varying the initial gas fraction is in good agreement with the observed dispersion of $\Delta_{\log M_{\mathrm{BH}}}=0.25-0.3$. We also note that our results are in good agreement with the $z=0 M_{\mathrm{BH}}-\sigma$ relation derived by Robertson et al. (2006c). We do not find any statistically significant difference between the 3:1, 1:1 and the complete merger sample, with all three samples resulting in virtually the same normalization coefficients and slopes for the $M_{\mathrm{BH}}-\sigma$ relation. The normalization coefficient steadily increases with increasing initial gas mass fraction. The dispersion within a sample with constant gas mass fraction is in general low, with the exception of the sample with the largest gas mass fraction. This can be taken as a further indication that the dispersion in the $M_{\mathrm{BH}}-\sigma$ relation is primarily driven by changing initial gas mass fractions. We conclude that the simulated $M_{\mathrm{BH}}-\sigma$ relation is overall robust and in agreement with the observed relation over a wide range of progenitor masses and initial gas fractions for both equal- and unequal-mass disk mergers.

Similarly, we perform a least-squares fit to the $M_{\mathrm{BH}}-$ $M_{*}$ relation as defined in Eq. 4, with the best fit parameters, parameter errors and dispersions for our samples listed in Table 5. We here define $M_{*}$ as the total mass of all luminous particles within the effective radius $r_{\mathrm{e}}$, 
including disk, bulge and newly formed stellar particles. This definition assumes that the merger remnant is a pure bulge system within this radius. We tested this assumption by performing a simple disk-bulge decomposition following the methodology outlined in Abadi et al. (2003). We calculated the orbital circularity parameter $\epsilon_{J}=J_{z} / J_{\text {circ }}(E)$ for all stellar particles within the effective radius, where $J_{z}$ is the z-component of the specific angular momentum and $J_{\text {circ }}(E)$ is the specific angular momentum expected for a corotating circular orbit with binding energy $E$. We found that the fraction of particles on circular orbits, as defined by $\epsilon_{J}>0.8$ was well below $1 \%$ for $1: 1$ merger remnants and at most $2 \%$ for 3:1 merger remnants. Thus, for $1: 1$ and 3:1 mergers the assumption that the remnants are bulge-dominated is valid, whereas for higher mass ratio mergers this method might induce some additional scatter due to an underlying surviving disk component in the final merger remnant (Naab \& Truillo 2006). In addition, we found that this definition for $M_{*}$ correlates reasonably well with total bulge mass as defined by the stellar bulge particles and finally this definition for $M_{*}$ is similar to the one employed by Di Matteo et al. (2008); Sijacki et al. (2007) and allows us to directly compare our results to theirs. Thus we adopt the simple definition of the total stellar mass within the effective radius as a proxy for the total bulge mass for all subsequent measurements of $M_{*}$.

The best fit value of the normalization coefficient $(c)$ for our total sample is in excellent agreement with the observations, but the derived slope $d$ is too steep compared to the observed sample. The too large value of the slope is a general problem in all our simulation samples as can be seen in Fig. [5. For low-mass stellar systems the simulations typically produce too low $\mathrm{BH}$ masses, whereas for the higher-mass stellar systems the $\mathrm{BH}$ mass is slightly overpredicted, thus producing a tilt in the simulated relation with respect to the observed relation. The derived dispersion in the simulated relation is marginally higher than the observed scatter, with the scatter being again primarily driven by variations in the initial gas mass fraction. Interestingly the final luminous mass within the effective radius remains virtually constant with changing initial gas mass fraction. In higher gas mass fraction simulations the lower initial stellar disk mass is compensated by higher star formation rates resulting in roughly the same final stellar mass. On the other hand, higher initial gas mass fractions produce larger final $\mathrm{BH}$ masses, thus resulting in vertical scatter in the $M_{\mathrm{BH}}-M_{*}$ relation as can be seen in Fig. 5], This also results in a systematic increase in the normalization coefficient with increasing gas mass fraction, with the slope remaining relatively constant. A plausible explanation for the discrepancy between the simulated and observed slopes of the $M_{\mathrm{BH}}-M_{*}$ relation lies in the employed stellar feedback model. A more aggressive stellar feedback including galactic winds (Springel \& Hernquist 2003; Oppenheimer \& Davé 2006) would primarily affect the low-mass stellar systems, reducing their final stellar mass, thus lowering the simulated slope and bringing it in better agreement with the observed slope. Indeed, both the cosmological studies by Di Matteo et al. (2008); Sijacki et al. (2007) found a better fit to the expected relations at the low mass end when they included a model for supernova-driven galactic winds in their simulations.
TABLE 5

Best Fit $M_{\mathrm{BH}}-M_{*}$ RELATION FOR 3:1 AND 1:1 MERGERS

\begin{tabular}{ccccc}
\hline \hline Sample & $\mathrm{N}$ & $\mathrm{c}$ & $\mathrm{d}$ & $\Delta_{\log M_{\mathrm{BH}}}$ \\
\hline Tot sample & 36 & $8.17 \pm 0.10$ & $1.40 \pm 0.07$ & 0.44 \\
3:1 sample & 18 & $8.04 \pm 0.11$ & $1.34 \pm 0.08$ & 0.47 \\
1:1 sample & 18 & $8.24 \pm 0.10$ & $1.41 \pm 0.10$ & 0.38 \\
S1-S2 20\% gas sample & 10 & $7.86 \pm 0.07$ & $1.34 \pm 0.05$ & 0.17 \\
S1-S2 40\% gas sample & 10 & $8.28 \pm 0.08$ & $1.45 \pm 0.06$ & 0.22 \\
S1-S2 80\% gas sample & 10 & $8.68 \pm 0.13$ & $1.36 \pm 0.12$ & 0.29 \\
Observed sample & 30 & $30.20 \pm 0.10$ & $1.12 \pm 0.06$ & 0.30 \\
\hline
\end{tabular}

${ }^{a}$ From Häring \& Rix (2004)

An alternative explanation for the discrepancy lies in the fact that the progenitors of lower mass galaxy mergers could have larger initial gas mass fractions compared to the higher mass merger progenitors. This would also effectively remove the tilt in the derived correlation bringing it in better agreement with the observed relation. Given these limitations in the stellar feedback model employed in our simulations we find that the final fit to the $M_{\mathrm{BH}}-M_{*}$ relation is adequate, with a well defined normalization and a slightly steeper slope compared to the observational relation.

\subsection{The accretion history of BHs in unequal mergers}

We now proceed to study the BH accretion and star formation histories for unequal-mass mergers with varying mass ratios. To this end we ran five mergers with mass ratios of $1: 1,2: 1,3: 1,4: 1$ and $6: 1$ on co-planar prograde orbits (G0). This orbital configuration was chosen in order to maximize the non-axisymmetric torques during the merger resulting in the most intensive bursts in $\mathrm{BH}$ accretion and star formation activity (Cox et al. 2008). All the progenitor galaxies had initial gas mass fractions of $20 \%$ with the primary progenitor having $v_{\text {vir }}=160 \mathrm{kms}^{-1}$ at the standard numerical resolution. The secondary galaxies were scaled down in mass and numerical resolution accordingly in order to maintain equal mass resolution per particle. The initial seed $\mathrm{BH}$ mass was set to $M_{\mathrm{BH} \text {,init }}=10^{5} M_{\odot}$ for all models. Again, the initial separation and pericentric distances are set to the mean of the virial radius and twice the mean of the scale radius respectively as detailed in 3.4

In Fig. 6] we show the evolution of the resulting star formation (top), $\mathrm{BH}$ accretion (middle) and $\mathrm{BH}$ mass (bottom) for the five mergers as a function of time. Increasing the mass ratio of the merger systematically lowers the peak star formation rate and increases the duration of star formation activity after the merger. For the highest mass ratio merger the star formation rate is virtually constant throughout the simulation with only a mild peak during the first passage (see also di Matteo et al. 2007; Bournaud et al.2007b). A similar evolution is seen in the $\mathrm{BH}$ accretion rates, with the $\mathrm{BH}$ accretion rates systematically peaking at higher rates with decreasing merger mass ratios. For the higher mass ratio mergers no well defined $\mathrm{BH}$ accretion peak is discernable, instead the $\mathrm{BH}$ accretion rates stay roughly constant on an elevated level for a prolonged time period compared to the pre-merger $\mathrm{BH}$ accretion rates. The evolution of the $\mathrm{BH}$ accretion rates are mirrored in the growth of the $\mathrm{BH}$ masses, with the final BH mass being systematically lower for increasing mass ratio of the merger. Furthermore, the slope of the $M_{\mathrm{BH}}$ growth as a function of time becomes shal- 


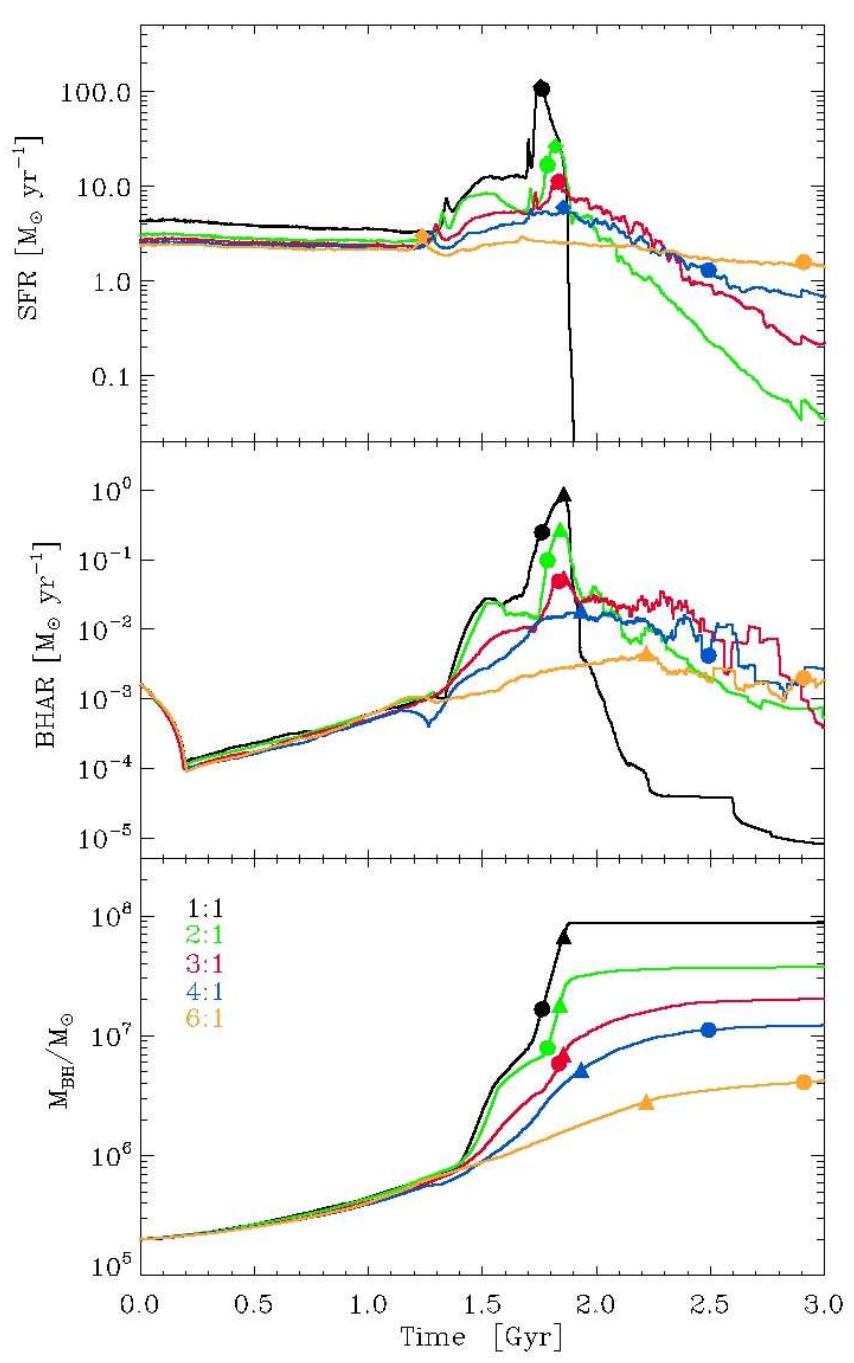

FIG. 6. - The total star formation rate (top), the total black hole accretion rate (middle) and the evolution of the total black hole mass (bottom) as a function of time for co-planar prograde (G0) 1:1 (black), 2:1 (green), 3:1 (red), 4:1 (blue) and 6:1 (orange) mergers. The filled circles indicate the time of the $\mathrm{BH}$ merger, the filled diamonds in the top panel and the filled triangles in the bottom two panels show the location of the maximum star formation and $\mathrm{BH}$ accretion peaks, respectively.

lower with increasing merger mass ratio. There is also a systematic delay in the time of the $\mathrm{BH}$ merger with increasing mass ratio, as indicated by the filled circles in Fig. 6. For the lower mass ratio mergers the peak of the $\mathrm{BH}$ activity (the filled triangles in Fig. 6) typically occurs shortly after the merging of the BHs, whereas for the higher mass ratio mergers the peak of the $\mathrm{BH}$ activity is not directly related to the merging time of the BHs.

In Fig. 7 we study the duration of the $\mathrm{BH}$ accretion and star formation activity as a function of merger mass ratio. We define a half-mass growth time $\Delta T_{1 / 2}$ during which half of the final $\mathrm{BH}$ mass and half of the total stellar mass is formed respectively. In both cases $\Delta T_{1 / 2}$ is centered on the peak of the corresponding activity, the $\mathrm{BH}$ accretion on the maximum of the $\mathrm{BH}$ accretion rate (the triangles in Fig. 6) and the star formation rate on the peak of the SFR marked with diamonds in Fig. 6. The resulting half-mass timescales are shown in the bottom panel of Fig. 7. The $\Delta T_{1 / 2} \mathrm{BH}$ is strongly cor-

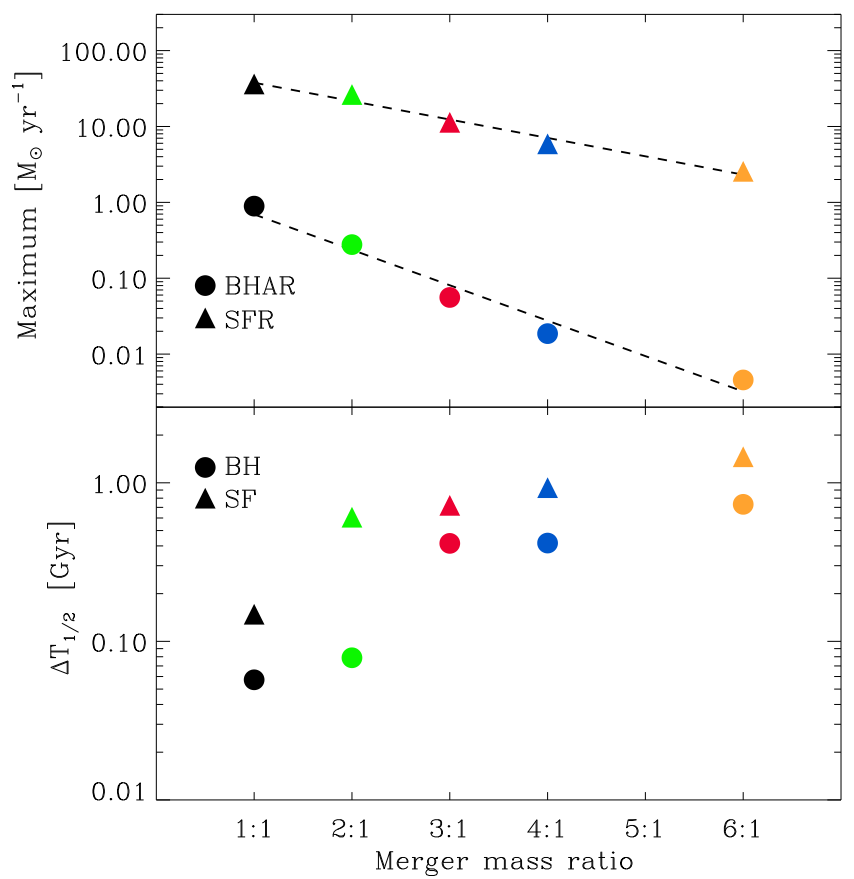

FIG. 7. - The maximum BH accretion and star formation rates and timescales as a function of merger mass ratio. The bottom panel shows the half mass growth time of the BHs (circles) and the stellar mass (triangles) centered on the maximum $\mathrm{BH}$ accretion and star formation rates, respectively. The top panel shows the corresponding peak $\mathrm{BH}$ accretion (circles) and star formation rates (triangles) during the corresponding half mass growth time $\Delta T_{1 / 2}$. The dashed line is the best resulting fit of Eq. 5 to the peak values.

related with the mass ratio of the merger. For 1:1 and 2:1 mergers the growth of the $M_{\mathrm{BH}}$ is very concentrated in time, with half of the final $\mathrm{BH}$ mass growth occurring in less than $100 \mathrm{Myr}$. For the 3:1 and 4:1 mergers $\Delta T_{1 / 2} \mathrm{BH} \sim 0.5 \mathrm{Gyr}$, with the $6: 1$ merger resulting in $\Delta T_{1 / 2 \mathrm{BH}} \sim 1 \mathrm{Gyr}$. In the lower mass ratio mergers a significant fraction of the gas is funnelled to the center during the merger, enabling the $\mathrm{BHs}$ to experience rapid Eddington limited growth (Eq. A2). For larger mass ratio mergers the tidally driven non-axisymmetric torques are weaker and a lower fraction of the gas reaches the center resulting in lower $\mathrm{BH}$ accretion rates and longer $\mathrm{BH}$ half-mass growth timescales.

The corresponding stellar half-mass timescales $\Delta T_{1 / 2_{\text {star }}}$ (triangles in the bottom panel Fig. 7) also show a clear correlation with the mass ratio of the merger. In the 1:1 merger half of the final stellar mass is formed in a short major burst lasting about $\Delta T_{1 / 2_{\text {star }}} \sim 0.15$ Gyr. This value is comparable to the star formation timescale derived by Cox et al. (2008), who calculated a full width at half maximum of FWHM $\sim 0.1$ Gyr for the star formation peak of a typical 1:1 merger. For higher mass-ratio mergers the resulting star formation timescales are $\Delta T_{1 / 2_{\text {star }}} \sim 0.7-1.0 \mathrm{Gyr}$, with the highest mass-ratio merger having the longest timescale of $\Delta T_{1 / 2}$ star $\sim 1.5 \mathrm{Gyr}$. In these cases it is not straightforward to relate our $\Delta T_{1 / 2}$ star to the burst timescales defined by Cox et al. (2008), as the higher mass ratio mergers have less well defined star formation peaks with the star formation distributed more evenly 
over time. However, the general trend that increasing the merger mass ratio results in increasingly longer star formation timescales agrees well with the results of Cox et al. (2008).

Finally we plot in the top panel of Fig. 7 the corresponding maximum black hole accretion and star formation rates during their respective half mass growth timescales. By defining the variable $q$ as the mass ratio between the primary and secondary component we can fit the logarithms of the peak $\mathrm{BH}$ accretion and star formation rates with the following linear relation

$$
\log \text { Maximum }\left[\mathrm{M}_{\odot} \mathrm{yr}^{-1}\right]=\mathrm{a}_{0}+\mathrm{a}_{1} \times \mathrm{q},
$$

where $a_{0}$ and $a_{1}$ are the inferred normalization and slope respectively. Both the maximum $\mathrm{BH}$ accretion rates and star formation rates are well fitted by Eq. 5] (dotted lines in Fig. (7) resulting in the following best fitting parameters respectively: $\left(a_{0, B H A R}=0.31, a_{1, B H A R}=-0.47\right)$ $\left(a_{0, S F R}=1.82, a_{1, S F R}=-0.24\right)$. The ratio of the peak $\mathrm{BH}$ accretion rate to the peak star formation rate is thus of the order of $\dot{M}_{\mathrm{BH} \text {,peak }} / \dot{M}_{\mathrm{SF} \text {,peak }} \sim 10^{-2}$. On the other hand, the ratio of the mean $\mathrm{BH}$ accretion rate to the mean star formation averaged over the whole simulation is closer to $\dot{M}_{\mathrm{BH}, \text { mean }} / \dot{M}_{\mathrm{SF} \text {, mean }} \sim 10^{-3}$, with this ratio varying systematically between $\dot{M}_{\mathrm{BH}, \text { mean }} / \dot{M}_{\mathrm{SF} \text {,mean }}=$ $2 \cdot 10^{-3}(1: 1$ merger $)$ and $\dot{M}_{\mathrm{BH}, \text { mean }} / \dot{M}_{\mathrm{SF}, \text { mean }}=0.2 \cdot 10^{-3}$ (6:1 merger). Interestingly, recent observations of $z \sim 2$ galaxies by Daddi et al. (2007b a ) also find indications of an universal ratio of $\dot{M}_{\mathrm{BH}} / \dot{M}_{\mathrm{SF}} \sim 10^{-3}$ and this is also expected from the observed $M_{\mathrm{BH}}-M_{*}$ relation. Naturally the normalizations and to some extent the slopes of the derived $\mathrm{BH}$ and SFR activity relations are dependent on the initial gas mass fraction and orbital geometry of the system. However, the derived ratios between the $\mathrm{BH}$ accretion and SF rates should be valid and we find in our simulations that for any given orbital configuration and initial gas mass fraction the ratio of the peak $\mathrm{BH}$ accretion and SF rates is $\sim 10^{-2}$, whereas the corresponding mean value is of the order of $\sim 10^{-3}$.

\subsection{Mixed E-Sp mergers with BHs}

We now turn our attention to mixed E-Sp mergers, in which a gas-poor early-type galaxy merges with a gasrich spiral disk galaxy. To this end we simulated to our knowledge for the first time a large sample of mixed E-Sp mergers. For early-type progenitors we use the merger remnants of $1: 1$ and 3:1 mergers discussed in 34.1 . whereas the progenitor disk galaxies are setup using the disk models discussed in 3.1 . Our mixed merger sample consisted of five 1:1 merger remnants that are merged with the primary disks of the original simulations (S1-ESp) and another five runs in which 3:1 merger remnants are merged with the corresponding secondary disks of the original simulations (S2-E-Sp). This sample of ten mixed mergers were run on the same G0 orbit as the original mergers. In addition, we simulated six E-Sp mergers of 1:1 and 3:1 merger remnants with primary and secondary disks respectively (G1-E-Sp and G2-E-Sp) with varying initial orientations and orbital geometries as indicated in Table 6. This results in a total sample of 16 mixed E-Sp mergers. The initial gas fraction was set to $20 \%$ in both the original simulations that produced the E-progenitors

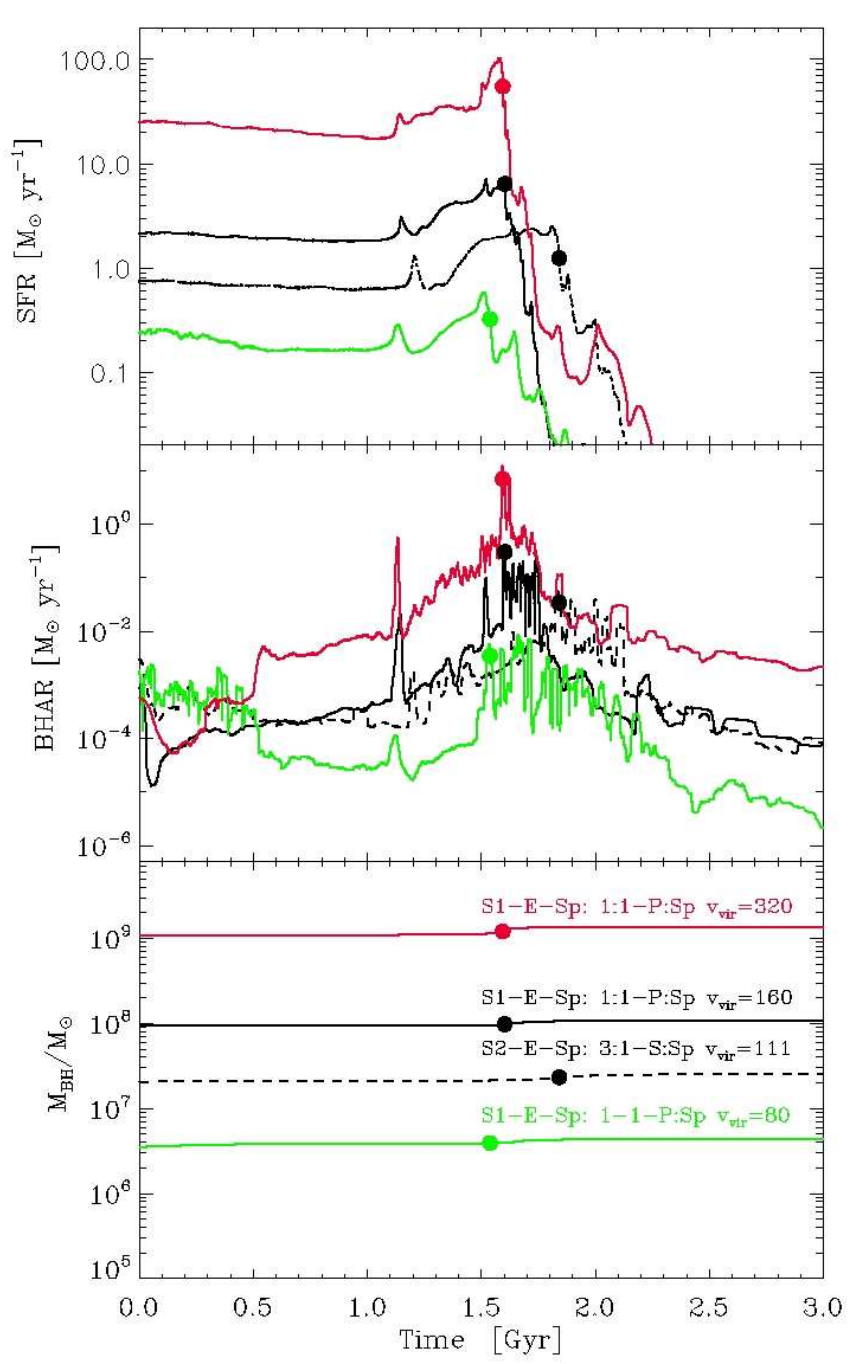

FIG. 8. - The total star formation rate (top), the total black hole accretion rate (middle) and the evolution of the total black hole mass (bottom) as a function of time for four E-Sp mixed mergers. The solid lines show mixed E-Sp mergers of 1:1 merger remnants with primary spiral disks and the dashed line shows a mixed E-Sp merger between a 3:1 merger remnant and a secondary spiral disk. The gas disks have initially $f_{\text {gas }}=20 \%$ and the virial velocities as indicated in the Figure. The filled circles indicate the time of merging of the BHs.

and in the disk progenitors. The black hole mass of the early-type progenitors is set by the result in the original simulation and is typically $M_{\mathrm{BH}} \sim 10^{6}-10^{9} M_{\odot}$, whereas the initial seed $\mathrm{BH}$ mass of the disk galaxies is always fixed at $M_{\mathrm{BH}}=10^{5} M_{\odot}$.

We plot the corresponding total star formation rates (top), the total $\mathrm{BH}$ accretion rates (middle) and the evolution of the total BH mass (bottom) in Fig. 8 for a sample of four mixed E-Sp mergers. The three solid lines show mergers between 1:1 merger remnants and primary disks and the dashed line a merger between a 3:1 merger remnant and a secondary disk, with the color scaling indicating the virial velocity of the disk progenitors. The combined star formation rate of the mixed E-Sp mergers is lower than in Sp-Sp (Fig. 3) mergers, due to the lower amount of cold gas available for star formation. The disk progenitor contains $f_{\text {gas }}=20 \%$ of gas initially, whereas the early-type progenitors typically have an initial gas 
TABLE 6

Mixed E-SP MERGERS

\begin{tabular}{|c|c|c|c|c|c|c|c|c|c|}
\hline Models & Progenitor 1 & $v_{\text {vir }, \operatorname{prog} 2}{ }^{a}$ & Mass ratio & Orbit & $f_{\text {gas,init }}$ & $N_{\text {disk,tot }}$ & $N_{\text {bulge,tot }}$ & $N_{\text {DM, tot }}$ & $N_{\text {sim }}$ \\
\hline S1-E-Sp & S1-1:1 & $50,80,160,320,500$ & $2: 1$ & G0 & 0.2 & 60000 & 30000 & 90000 & 5 \\
\hline S2-E-Sp & S2-3:1 & $35,55,111,222,347$ & $4: 1$ & G0 & 0.2 & 33334 & 16666 & 50000 & 5 \\
\hline G1-E-Sp & G1-1:1 & 160 & $2: 1$ & G7, G10, G13 & 0.2 & 60000 & 30000 & 90000 & 3 \\
\hline G2-E-Sp & G2-3:1 & 111 & $4: 1$ & G4, G10, G14 & 0.2 & 33334 & 16666 & 50000 & 3 \\
\hline
\end{tabular}

${ }^{a}$ All virial velocities in $\mathrm{kms}^{-1}$
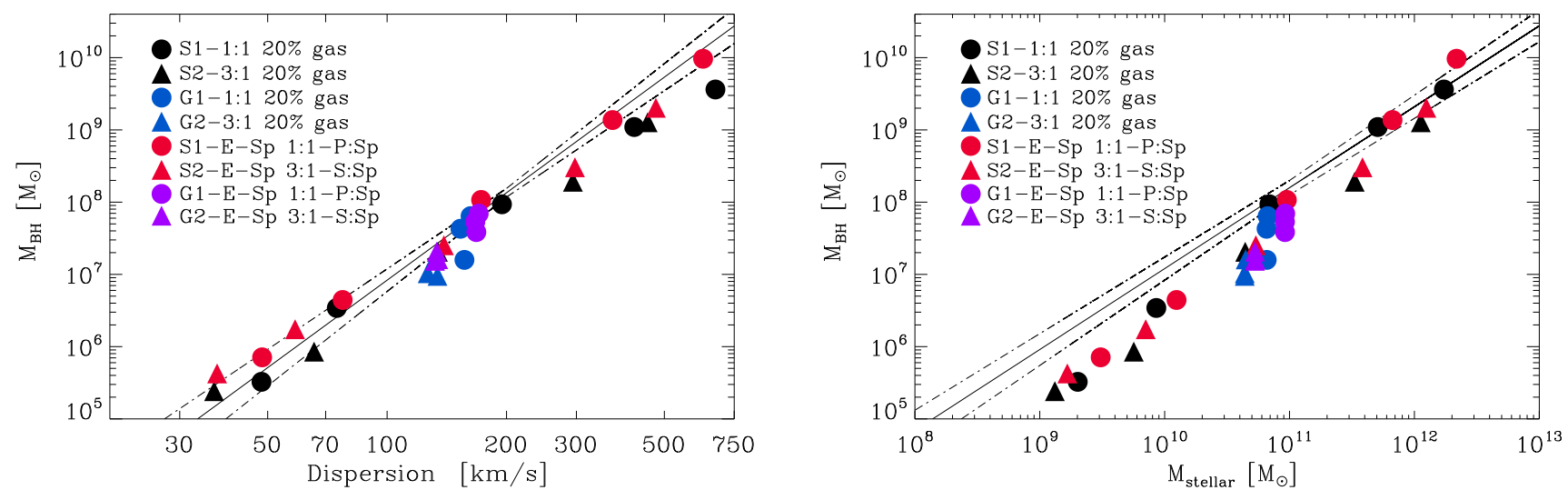

Fig. 9.- The $M_{\mathrm{BH}}-\sigma$ (left) and $M_{\mathrm{BH}}-M_{*}$ (right) relations for our sample of mixed E-Sp mergers. The merger remnants of the original 1:1 and 3:1 mergers are shown by black and blue filled circles and triangles for the $\mathrm{S}$ and $\mathrm{G}$ merger series, respectively (Table 3 ). The mixed merging of 1:1 merger remnants with primary spiral disks with $f_{\text {gas }}=20 \%$ (1:1-P:Sp) are shown with filled red (S1-series) and purple (G1-series) circles. The mixed merging of 3:1 merger remnants with secondary spiral disks with $f_{\text {gas }}=20 \%(3: 1-\mathrm{S}: \mathrm{Sp})$ are shown with filled red (S2-series) and purple (G2-series) triangles. The lines show the observed relations with errors by Tremaine et al. (2002) (left panel) and Häring \& Rix (2004) (right panel), respectively.

TABLE 7

Best Fit $M_{\mathrm{BH}}-\sigma$ RELATION FOR E-E ANd E-Sp MERGERS

\begin{tabular}{ccccc}
\hline \hline Sample & $\mathrm{N}$ & $a$ & $b$ & $\Delta_{\log M_{\mathrm{BH}}}$ \\
\hline Progenitor sample & 16 & $7.83 \pm 0.04$ & $3.53 \pm 0.11$ & 0.16 \\
E-Sp Mixed sample & 16 & $8.03 \pm 0.04$ & $3.55 \pm 0.12$ & 0.13 \\
E-E Remerger sample & 16 & $8.13 \pm 0.05$ & $3.41 \pm 0.10$ & 0.18 \\
\hline
\end{tabular}

mass fraction of $f_{\text {gas }} \sim 5 \%$, with typically only $\sim 1 \%$ of this gas being cold and dense. After the merger of the E-Sp galaxies the star formation rate declines rapidly in all the merger remnants. The black hole accretion rates are somewhat lower than in Sp-Sp mergers, but still relatively high due to the large amount of available gas in the central regions of the merger remnants originating from the disk progenitors. The $\mathrm{BH}$ accretion rates show a clear peak, both during the time of the first passage and at the time of final coalescence. We define a $\mathrm{BH}$ mass growth factor as $f_{\mathrm{BH}, \text { insitu }}=\Delta M_{\mathrm{BH} \text {,insitu }} / M_{\mathrm{BH}, \text { final }}$, which gives the ratio of $\mathrm{BH}$ mass growth due to gas accretion during the simulation with respect to the final $\mathrm{BH}$ mass. Quantitatively, the fraction of the $\mathrm{BH}$ mass that accumulates by gas accretion during the mixed mergers is in the range of $f_{\mathrm{BH} \text {,insitu }} \sim 20-50 \%$, with typical mean values of $f_{\mathrm{BH}, \text { insitu }} \sim 30 \%$. Thus in most cases the majority of the final $\mathrm{BH}$ mass is still contributed by the massive seed BHs residing in the early-type progenitors.

The $M_{\mathrm{BH}}-\sigma$ (left panel) and $M_{\mathrm{BH}}-M_{*}$ (right panel) relations for our mixed E-Sp merger (red and purple symbols) sample together with the E-type progenitor population (black and blue symbols) is plotted in Fig. 9. All the progenitor disk galaxies have initial seed $\mathrm{BH}$ masses of $M_{\mathrm{BH}}=10^{5} M_{\odot}$ with the velocity dispersion $\sigma$ depend- ing on the bulge mass of the progenitor galaxy. Thus the $\mathrm{BH}$ of the disk galaxies are not initially on the $M_{\mathrm{BH}}-\sigma$ relation and they are also not plotted in Fig. 9, Using a least-squares fit and Bootstrap technique we derive the best fitting parameters for the $M_{\mathrm{BH}}-\sigma$ relation of our mixed merger and progenitor samples (see Table 7). For the mixed mergers the normalization parameter $a$ is 0.2 dex higher than for the Sp-Sp progenitor sample, but 0.1 dex lower than for the E-E mergers. The combined insitu growth due to gas accretion and merging of the $\mathrm{BHs}$ thus result in final BH masses that are very similar to the ones derived in both the Sp-Sp (Fig. 5) and E-E mergers (Fig. 11). The mixed merging of E-Sp galaxies seems to maintain the slope $b$ of the $M_{\mathrm{BH}}-\sigma$ relation compared to the progenitor sample and even slightly reduces the dispersion. The mixed mergers primarily increase the final $\mathrm{BH}$ mass, with smaller changes in the final velocity dispersion compared to the initial dispersion of the early-type progenitors. Overall the slope and dispersion are lower than the observed Tremaine et al. (2002) values of $b=4.02 \pm 0.32, \Delta_{\log M_{\mathrm{BH}}}=0.25-0.3$. This is primarily due to the fact that all of the original mergers and disk progenitors had initial gas mass fractions of $20 \%$. Including mixed mergers of merger remnants and disks with higher initial gas mass fractions would increase the normalization, slope and scatter in the derived $M_{\mathrm{BH}}-\sigma$ relation (see Table 44). The mixed mergers undergo a similar phase of strong self-regulated $\mathrm{BH}$ growth as the Sp-Sp mergers, allowing the BH feedback to regulate the final $\mathrm{BH}$ mass in agreement of the $M_{\mathrm{BH}}-\sigma$ relation. Thus we conclude that the $M_{\mathrm{BH}}-\sigma$ relation should remain relatively stable even after several generations of mixed E-Sp mergers. 
TABLE 8

Best fit $M_{\mathrm{BH}}-M_{*}$ RELATION FOR E-SP AND E-E MERGERS

\begin{tabular}{ccccc}
\hline \hline Sample & $\mathrm{N}$ & $\mathrm{c}$ & $\mathrm{d}$ & $\Delta_{\log M_{\mathrm{BH}}}$ \\
\hline Progenitor sample & 16 & $7.78 \pm 0.07$ & $1.35 \pm 0.05$ & 0.21 \\
E-Sp Mixed sample & 16 & $7.83 \pm 0.05$ & $1.39 \pm 0.07$ & 0.16 \\
E-E Remerger sample & 16 & $7.86 \pm 0.05$ & $1.38 \pm 0.04$ & 0.17 \\
\hline
\end{tabular}

Finally, we also perform a least-squares fit to the $M_{\mathrm{BH}}-M_{*}$ (Eq. 4) relation for our mixed E-Sp sample with the best fit parameters listed in Table 8 . The mixed merging of an E-Sp galaxy population effectively maintains the original $M_{\mathrm{BH}}-M_{*}$ relation of the progenitor sample, even slightly reducing the intrinsic dispersion in the relation. The self-regulated stellar and $\mathrm{BH}$ feedback models regulate the star formation process and $\mathrm{BH}$ mass growth in mixed mergers similarly to $\mathrm{Sp}-\mathrm{Sp}$ mergers thus ensuring that the derived original $M_{\mathrm{BH}}-M_{*}$ relation is maintained. We thus conclude that even repeated generations of mixed E-Sp mixed mergers should not greatly affect the observed $M_{\mathrm{BH}}-M_{*}$ relation.

\subsection{E-E remergers with BHs}

Finally, we studied E-E mergers including BH feedback by remerging a sample of our merger remnants produced by the 3:1 and 1:1 mergers discussed in 4.1. We set up and ran five equal-mass remergers of our S1-1:1 (Table (3) merger remnants (S1-E-E), with the initial progenitor disks of the original simulations having virial velocities in the range $v_{\mathrm{vir}}=50-500 \mathrm{kms}^{-1}$. In addition, we remerged five of our 1:1 merger remnants with the corresponding set of 3:1 merger remnants (S2-E-E), resulting in a mass ratio of 3:2 (see Table 9). All of the mergers were run on identical orbits to the original mergers and the initial gas fraction of the progenitors for the original simulations was fixed at $20 \%$. In addition to the ten S-series remergers that were run on the G0 orbit, we simulated three E-E mergers of 1:1 merger remnants (G1-E-E) and three E-E mergers of 3:1 merger remnants (G2-E-E) with varying initial orientations and orbital geometries as indicated in Table 9, thus resulting in a total sample of $16 \mathrm{E}-\mathrm{E}$ remergers.

In Fig. 10] we plot the star formation rate (top), the $\mathrm{BH}$ accretion rate (middle) and the evolution of the $\mathrm{BH}$ mass (bottom) for three 1:1-3:1 E-E remergers (solid lines) and one 1:1-1:1 E-E remerger (dashed line). Here the coloring indicates the virial velocities of the primary galaxies in the original simulations, $v_{\mathrm{vir}, \mathrm{p}}=80 \mathrm{kms}^{-1}$ (green), $v_{\text {vir }, \mathrm{p}}=160 \mathrm{kms}^{-1}$ (black) and $v_{\mathrm{vir}, \mathrm{p}}=320 \mathrm{kms}^{-1}$ (red). The initial star formation rates are generally very low due to the low gas fractions of $f_{\text {gas }} \sim 1-5 \%$ and $f_{\text {gas }} \sim 5-10 \%$ for the 1:1 and 3:1 merger remnant Eprogenitors, respectively. The initial gas fraction directly depends on the strength of the initial interaction that gave rise to the merger remnants used as progenitors for the E-E remergers. Increasing the masses of the progenitors and using more direct planar orbits (G0) produces more violent initial encounters, thus decreasing $f_{\text {gas. }}$. In addition, most of the gas is hot with low densities and the fraction of cold star-forming gas is typically below $\lesssim 1 \%$ of the total gas mass. As can be seen in Fig. 10 the star formation is very effectively terminated shortly after the merging of the E-E progenitors on comparable timescales to 1:1 Sp-Sp mergers and thus more efficiently than in 3:1

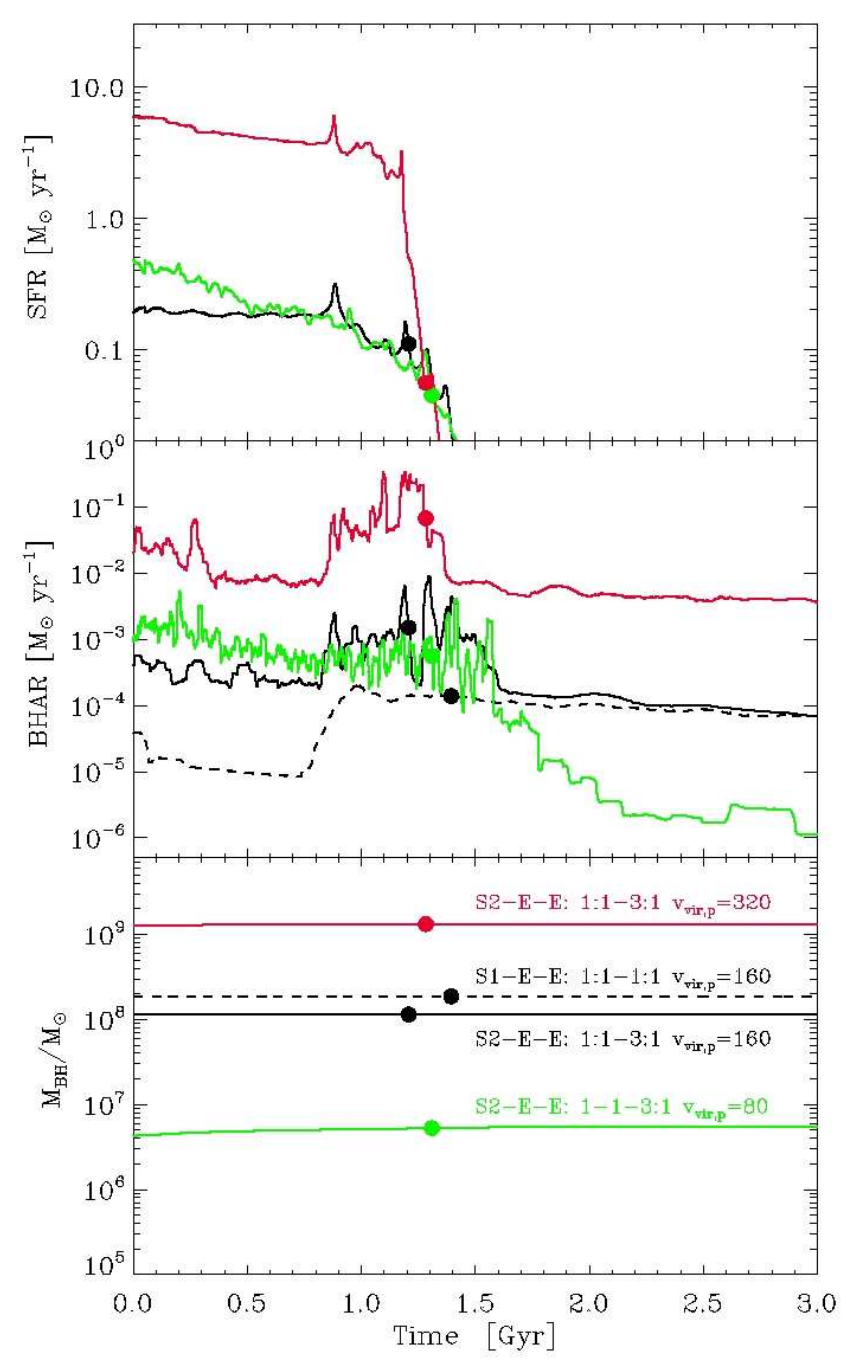

Fig. 10.- The total star formation rate (top), the black hole accretion rate (middle) and the evolution of the total black hole mass (bottom) as a function of time for four E-E remergers. The solid lines show remergers between 1:1 and 3:1 merger remnants and the dashed line shows a E-E remerger between two 1:1 merger remnants. The virial velocities of the primary galaxies in the original simulations are as indicated in the Figure. The filled circles indicate the time of merging of the BHs.

Sp-Sp and mixed E-Sp mergers. For the 1:1-1:1 E-E remerger the star formation rate is zero throughout the simulation as the star formation was already completely terminated in the original 1:1 merger that served as a progenitor galaxy for the E-E merger (see Figs. 3 and 6).

The black hole accretion rates strongly correlate with the available amount of gas in the central regions and are thus typically one to two orders of magnitude lower for dry E-E mergers compared to the mixed E-Sp and Sp-Sp mergers. This is also reflected in the total $\mathrm{BH}$ mass growth plots (Fig. 10) that remain virtually flat throughout the simulations. The growth of the final $\mathrm{BH}$ mass is primarily due to the merging of the $\mathrm{BHs}$ with the in-situ growth of the $\mathrm{BH}$ mass through gas accretion being relatively low. The exact fraction of $\mathrm{BH}$ mass grown in-situ $\left(f_{\mathrm{BH}, \text { insitu }}\right)$ due to gas accretion during the simulation with respect to the final $\mathrm{BH}$ mass, depends on the amount of available gas initially. For remerging 
TABLE 9

E-E REMERGERS

\begin{tabular}{|c|c|c|c|c|c|c|c|c|c|}
\hline Models & Progenitor 1 & Progenitor 2 & Mass ratio & Orbit & $f_{\text {gas, init }}$ & $N_{\text {disk, tot }}$ & $N_{\text {bulge,tot }}$ & $N_{\mathrm{DM}, \text { tot }}$ & $N_{\mathrm{sim}}$ \\
\hline S1-E-E & S1-1:1 & S1-1:1 & $1: 1$ & G0 & 0.2 & 80000 & 40000 & 120000 & 5 \\
\hline S2-E-E & S1-1:1 & $\mathrm{S} 2-3: 1$ & $3: 2$ & G0 & 0.2 & 66667 & 33333 & 100000 & 5 \\
\hline G1-E-E & G1-1:1 & G1-1:1 & $1: 1$ & G7, G10, G13 & 0.2 & 80000 & 40000 & 120000 & 3 \\
\hline G2-E-E & G2-3:1 & G2-3:1 & $1: 1$ & G4, G10, G14 & 0.2 & 26666 & 53334 & 80000 & 3 \\
\hline
\end{tabular}
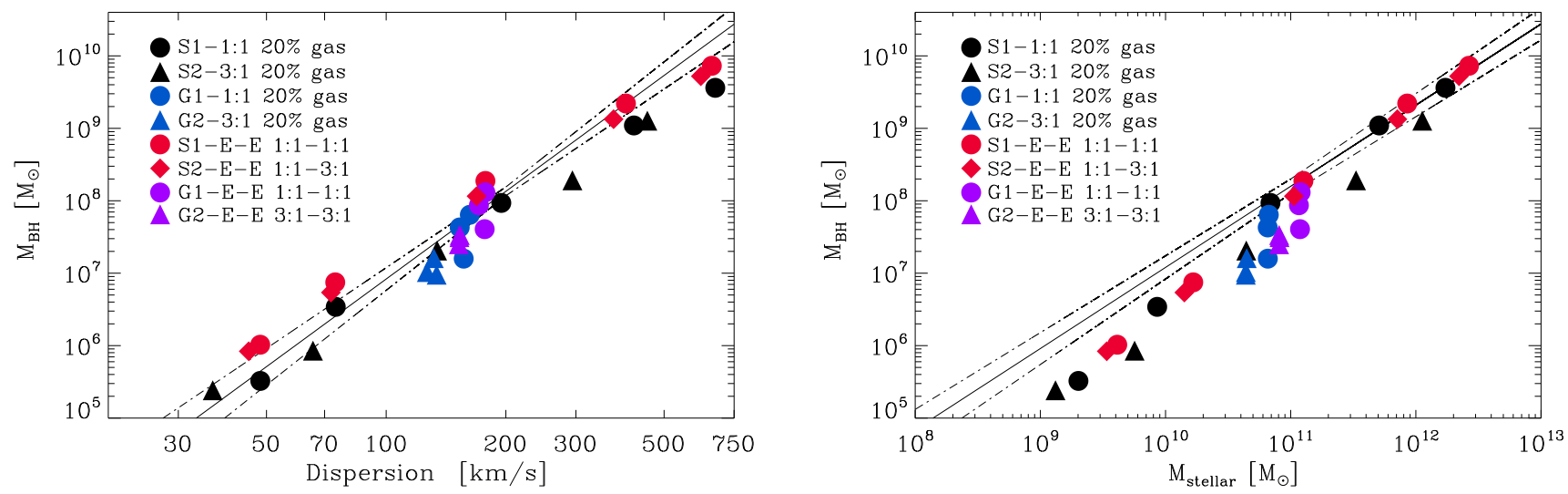

Fig. 11. - The $M_{\mathrm{BH}}-\sigma$ (left) and $M_{\mathrm{BH}}-M_{*}$ (right) relations for our sample of E-E remergers. The merger remnants of the original 1:1 and 3:1 mergers are shown by black and blue filled circles and triangles for the S and G merger series, respectively (Table 3). The remerging of 1:1-1:1 merger remnants are shown with filled red (S1-series) and purple (G1-series) circles, the remerging of 1:1-3:1 merger remnants (S2-series) are shown with red diamond symbols and finally the remerging of 3:1-3:1 remnants (G2-series) by purple triangles. The lines show the observed relations with errors by Tremaine et al. (2002) (left panel) and Häring \& Rix (2004) (right panel), respectively.

of $1: 1$ merger remnants this fraction is typically very low at $f_{\mathrm{BH}, \text { insitu }}<1 \%$, whereas for the remerging of 1:1-3:1 merger remnants the fraction is higher at $f_{\mathrm{BH}, \text { insitu }} \sim$ $10 \%$, with the highest fraction of $f_{\mathrm{BH}, \text { insitu }} \sim 20 \%$ produced for remerging of 3:1-3:1 merger remnants, as expected. There is relatively large scatter in the $f_{\mathrm{BH}, \text { insitu }}$ fraction as a function of the orbital configuration and the initial masses of the progenitor galaxies primarily set by the corresponding scatter in the initial gas mass fractions of the progenitor galaxies. All the fractions above are estimates for only first generation remergers, with the $f_{\mathrm{BH}, \text { insitu }}$ ratio rapidly decreasing with increasing generations of remergers. The same is true for the star formation, E-E remerging effectively quenches the star formation (Fig. 10) and already a second generation of dry merging is expected to show very weak, if any, signatures of residual star formation.

In Fig. 11 we plot the $M_{\mathrm{BH}}-\sigma$ (left panel) and the $M_{\mathrm{BH}}-M_{*}$ (right panel) relations for our E-E remerger (red and purple symbols) sample together with the progenitor population (black and blue symbols). The best fitted $M_{\mathrm{BH}}-\sigma$ parameters together with the $1 \sigma$ uncertainties for our E-E remerger sample are listed in Table 7. The normalization parameter $a$ is 0.3 dex higher for the E-E remerger sample compared to the progenitor sample and 0.1 dex higher compared to the mixed E-Sp sample. However the slope $b$ is marginally shallower compared to both the progenitor and mixed merger samples. The EE remerging of the merger remnants increases the final $\mathrm{BH}$ mass, but leaves the velocity dispersion more or less unchanged, resulting in an increase of $a$. The relative increase in $M_{\mathrm{BH}}$ is slightly larger for low-mass systems compared to higher-mass systems, resulting in a slightly lower slope $b$. This is especially true for the remergers on the G0 orbit, whereas remergers on other orbits (the purple G-series in Fig. 11) also increase slightly the velocity dispersion of the resulting merger remnants (see also Robertson et al. 2006b). The overall lower value of the slope $b$ is again due to the fact that only E-E mergers of progenitors with initial gas fractions of $f_{\text {gas }}=20 \%$ in the original simulations were included in constructing the E-E sample. We thus conclude that dry E-E remerging typically increases the normalization parameter $a$ and lowers the slope $b$ slightly, but a single generation of dry remerging is not able to destroy the $M_{\mathrm{BH}}-\sigma$ relation initially generated by gas-rich mergers (see also Robertson et al. 2006b). E-E remerging also increases slightly the dispersion in the fitted relation, but the variation in the initial gas fraction still remains the dominant source of scatter. Unlike Sp-Sp and mixed E-Sp mergers the gas-poor E-E mergers only experience a weak selfregulatory $\mathrm{BH}$ growth phase, with this phase missing altogether for the gas-poorest E-E mergers. This lack of the self-regulating feedback phase can thus explain some of the larger scatter in the final E-E remerger $M_{\mathrm{BH}}-\sigma$ relation.

We also perform a least-squares fit to the $M_{\mathrm{BH}}-M_{*}$ (Eq. 4) relation for both the E-E remerger sample and the progenitor sample with the best fit parameters, parameter errors and dispersions listed in Table 8 . Dry E-E remerging effectively maintains the normalization coefficient $(c)$ and slope $(d)$ of the progenitor sample, with a very minor increase of both parameters that lie within the $1 \sigma$ uncertainties of the corresponding progenitor parameters. Furthermore dry remerging roughly maintains the dispersion derived for the progenitor population. This is expected since the in-situ growth from gas of both the $\mathrm{BH}$ and stellar mass are very low dur- 

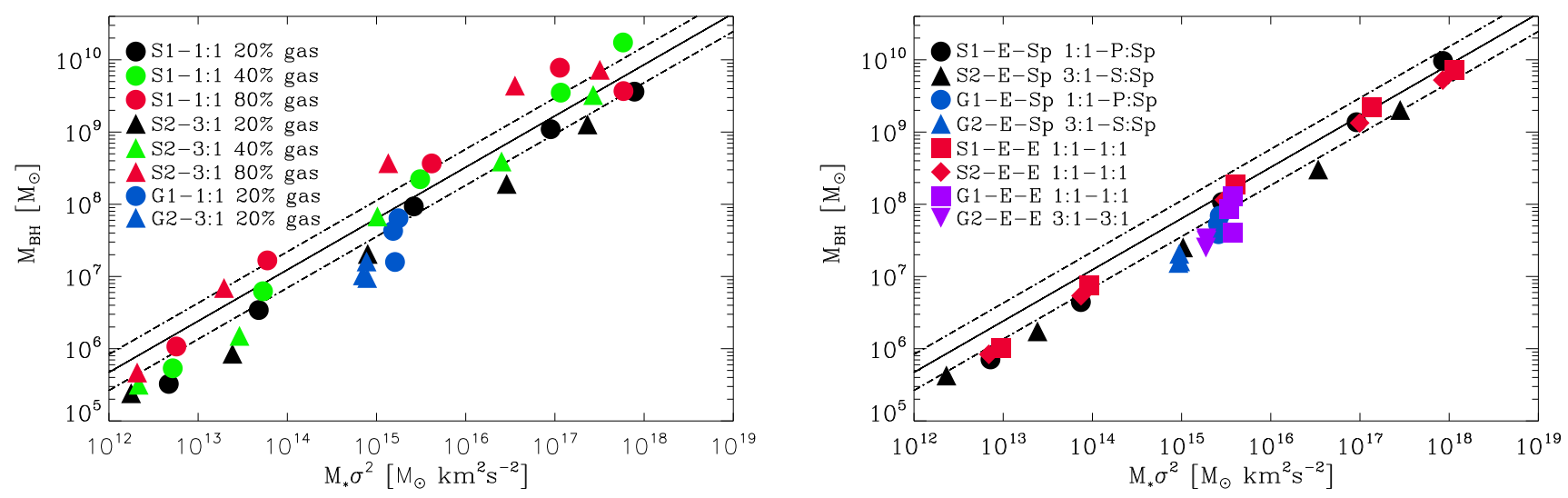

FIG. 12.- The binding energy correlation, which is statistically equivalent to the BHFP. In the left panel we plot the correlation for our complete sample of 3:1 (triangles) and 1:1 (circles) mergers, with the colors indicating variations in the initial gas mass fractions (black, green, red) and in the orbital geometries (blue). In the right panel we plot the corresponding relation for our mixed E-Sp (circles and triangles) and the E-E remerger sample (squares, diamonds and downward pointing triangles). The lines show the observed relation with scatter from the compilation of Hopkins et al. (2007a)

TABLE 10

Best Fit BINDING ENERGy RELATION FOR 3:1, 1:1, E-Sp AND E-E MERGERS

\begin{tabular}{ccccc}
\hline \hline Sample & $\mathrm{N}$ & $\mathrm{e}$ & $\mathrm{f}$ & $\Delta_{\log M_{\mathrm{BH}}}$ \\
\hline 3:1 sample & 18 & $8.05 \pm 0.10$ & $0.79 \pm 0.04$ & 0.41 \\
1:1 sample & 18 & $8.15 \pm 0.08$ & $0.81 \pm 0.05$ & 0.32 \\
S1-S2 20\% gas sample & 10 & $7.85 \pm 0.05$ & $0.76 \pm 0.03$ & 0.14 \\
S1-S2 40\% gas sample & 10 & $8.22 \pm 0.06$ & $0.84 \pm 0.03$ & 0.17 \\
S1-S2 80\% gas sample & 10 & $8.54 \pm 0.12$ & $0.79 \pm 0.06$ & 0.28 \\
E-Sp Mixed sample & 16 & $7.91 \pm 0.06$ & $0.77 \pm 0.03$ & 0.15 \\
E-E Remerger sample & 16 & $7.98 \pm 0.05$ & $0.76 \pm 0.02$ & 0.18 \\
Total sample & 68 & $8.04 \pm 0.05$ & $0.79 \pm 0.02$ & 0.31 \\
Observed sample $^{a}$ & 38 & $8.23 \pm 0.06$ & $0.71 \pm 0.06$ & 0.25 \\
\hline
\end{tabular}

${ }^{a}$ From Hopkins et al. (2007a)

ing the simulation. For the limiting case of no available gas and no in-situ growth of the $\mathrm{BH}$ and stellar mass, the original $M_{\mathrm{BH}}-M_{*}$ relation would be perfectly conserved. Thus we expect that increasing generations of E-E mergers would not substantially increase the scatter in the observed $M_{\mathrm{BH}}-M_{*}$ relation. The discrepancy between the derived $M_{\mathrm{BH}}-M_{*}$ parameters for the E-E remergers and the observed parameters would be remedied by remerging merger remnants with higher initial gas mass fractions (increases $c$ ) and by employing more effective stellar feedback models (lowers $d$ ) as was already discussed in 4.1

\subsection{The Black Hole fundamental plane}

Recent studies by Hopkins et al. (2007a,b) have shown that the well established $M_{\mathrm{BH}}-\sigma$ and $M_{\mathrm{BH}}-M_{*}$ relations are only two projections of a more fundamental underlying relation. This so called Black Hole Fundamental plane (BHFP) is defined as $M_{\mathrm{BH}} \sim \sigma^{3.0 \pm 0.3} R^{0.43 \pm 0.19}$ or $M_{\mathrm{BH}} \sim M_{*}^{0.54 \pm 0.17} \sigma^{2.2 \pm 0.5}$ and is analogous to the fundamental plane derived for elliptical galaxies (Hopkins et al. 2007a, b). A statistically equivalent formulation of the BHFP is given by the binding energy correlation, which relates the total binding energy of the bulge $\left(E \sim M_{*} \sigma^{2}\right)$ to the mass of the the BH. Recent observations by Aller \& Richstone (2007); Barway \& Kembhavi (2007) have shown that the bulge gravitational binding energy as traced by the stellar light profile correlates strongly with the $\mathrm{BH}$ mass.

Figure 12 shows the binding energy correlation for our 3:1 and 1:1 disk merger sample (left panel) and for the mixed E-Sp and dry E-E mergers (right panel), respectively, together with the observed relation from the compliation of Hopkins et al. (2007a). We perform a leastsquares fit to the data using the equation below

$$
\log \left(M_{\mathrm{BH}} / M_{\odot}\right)=e+f \log \left(M_{*} \sigma^{2} /\left(M_{0} \sigma_{0}^{2}\right)\right),
$$

where $e$ and $f$ are the normalization coefficient and slope, respectively, and $M_{0}=10^{11} M_{\odot}$ and $\sigma_{0}=200 \mathrm{~km} / \mathrm{s}$ are the normalization values. The best values of $e$ and $f$, together with the dispersion for the various subsamples are given in Tab. 10. We do not find any statistically significant difference between the $3: 1$ and 1:1 merger samples. For both samples the derived normalizations and slopes are very similar and in good agreement with the observed relation. Varying the initial gas mass fraction produces more pronounced differences, with a systematically higher normalization coefficient for increasing initial gas mass fractions. However, the slope remains constant within the error bars with changing initial gas mass fractions. The mixed E-Sp and dry E-E merger samples generally maintain the slope of the progenitor sample, with a slight increase in the normalization. Finally, combining all the merger samples together produces a final relation in reasonably good agreement with the observed relation and with a similar dispersion to the observed sample. The tightness of the derived correlation indicates that the final $\mathrm{BH}$ mass in all the merger samples is primarily set by the depth of the gravitational potential well.

We conclude that the bulge binding energy relation provides a robust predictor for the $\mathrm{BH}$ mass over a wide range of progenitor masses and initial gas fractions for both unequal- and equal-mass disk mergers, mixed E-Sp mergers and E-E mergers. The dispersion in the bulge binding energy correlation is similar in size to the dispersion in the $M_{\mathrm{BH}}-\sigma$ relation and both these methods should be preferred over the $M_{\mathrm{BH}}-M_{*}$ relation that produces significantly larger scatter (see Aller \& Richstone 2007 for a discussion). 


\section{SUMMARY AND DISCUSSION}

In this paper we have studied galaxy mergers with $\mathrm{BH}$ feedback starting with equal- and unequal-mass disk progenitors, mixed disk-elliptical progenitors and ellipticalelliptical progenitors. Extending on detailed previous studies of equal-mass disk mergers (e.g. Robertson et al. 2006a) our merger sample includes 18 unequal-mass disk mergers, 16 mixed E-Sp mergers and 16 dry E-E mergers in addition to 18 equal-mass mergers resulting in a total sample of 68 mergers. The simulations include radiative cooling, star formation and black holes with their associated feedback processes as described in Springel et al. (2005b).

We study the termination of star formation in merger simulations including $\mathrm{BH}$ feedback. We find that the termination of star formation by $\mathrm{BH}$ feedback is significantly less important for unequal-mass disk mergers compared to equal-mass disk mergers (Springel et al. 2005a). The timescale for star formation termination systematically increases with increasing progenitor mass ratios. Similarly, a systematic increase is seen in the half-mass growth timescales of the $\mathrm{BHs}$, with this timescale varying from $\sim 0.1$ Gyr for equal-mass mergers to $\sim 1$ Gyr for 6:1 mergers. This systematic trend can be used as input in modeling $\mathrm{BH}$ accretion more realistically in semi-analytic galaxy formation models (e.g. Croton et al. 2006). For mass-ratios of $3: 1$ and higher mergers with $\mathrm{BH}$ feedback are unable to completely quench the star formation, with the merger remnants showing star formation rates roughly on the pre-merger level even 1 Gyr after completion of the merger.

In mixed E-Sp mergers and dry E-E mergers the star formation and $\mathrm{BH}$ accretion rates are in general low due to the low cold gas mass fraction. The star formation is efficiently terminated in mixed E-Sp mergers due to the presence of the fully grown super-massive $\mathrm{BH}$ in the E-progenitor, with lower mass ratio mergers (2:1) again resulting in shorter termination timescales compared to higher mass ratio mixed (4:1) mergers. Finally, dry E-E mergers with two super-massive BHs are extremely efficient in terminating any residual star formation in the progenitors. Remergers of 1:1 merger remnants show typically no star formation, whereas remergers of lower mass ratio merger remnants can show star formation on the level of a few solar masses per year that is rapidly terminated in the merger. This implies that already a second generation of dry merging is expected to show very weak, if any, signatures of residual star formation.

We show that in addition to $1: 1$ disk mergers (Di Matteo et al. 2005) also 3:1 unequal-mass disk mergers, mixed E-Sp mergers and dry E-E mergers reproduce the observed $M_{\mathrm{BH}}-\sigma$ relation, with the dominant source of scatter arising from variations in the initial gas mass fractions of the galaxies. The 3:1 unequal-mass mergers require $\mathrm{BH}$ repositioning, in particular at low numerical resolution, which ensures that the final $\mathrm{BH}$ quickly resettles in the central gas disk after the merging of the BHs. In addition, the normalization of the observed $M_{\mathrm{BH}}-M_{*}$ relation is also well fitted in our simulation sample, but with the resulting slope being slightly steeper compared to the observed slope. This discrepancy might be resolved by employing a more aggressive supernova-driven wind feedback, as in recent self-consistent cosmological simulations (Sijacki et al. 2007; Di Matteo et al. 2008) or by the fact the progenitors of lower mass galaxy mergers have larger initial gas mass fractions compared to high mass galaxy mergers. Finally, our complete merger sample fits well the observed bulge binding energy correlation with black hole mass, $M_{\mathrm{BH}}-M_{*} \sigma^{2}$, which is statistically equivalent to the Black Hole Fundamental plane (Hopkins et al. 2007a, b) . The tightness of this correlation indicates that the final $\mathrm{BH}$ mass in all the merger samples is ultimately set by the depth of the gravitational potential well.

The remarkable robustness of the simulated $M_{\mathrm{BH}}-\sigma$, $M_{\mathrm{BH}}-M_{*}$ and $M_{\mathrm{BH}}-M_{*} \sigma^{2}$ relations for an extremely wide range of initial galaxy properties implies that the Springel et al. (2005b) effective BH feedback model selfregulates the mass growth of the $\mathrm{BHs}$ in accordance with the observed relations for a very wide range of merger progenitors. However, in addition it is important to study the detailed properties of merger remnants including $\mathrm{BH}$ feedback. It has been shown that the stellar orbits, e.g. the relative population of box and tube orbits, in merger remnants are affected by the amount of gas driven to the center Barnes \& Hernquist 1996; Jesseit et al. 2005, 2007). Here, even relatively small amounts of gas $(\sim 5 \%)$ can influence higher order photometric and kinematical properties of the remnants as a whole (Naab et al. 2006a). As efficient feedback from black holes reduces the central gas content in first generation disk merger remnants, future mergers will be less dissipative and favor the formation of triaxial stellar systems dominated by box orbits (Jesseit et al. 2005; Naab et al. 2006a). In addition, we studied in Burkert et al. (2007) the intrinsic anisotropy $\delta$ and flattening $\epsilon$ of simulated merger remnants with and without BH feedback. Here, an equally good fit to ellipticals observed by SAURON (Emsellem et al. 2004; Cappellari et al. 2006) was found in both models that included BH feedback and models without BHs. However, binary disk mergers and their descendants were shown to preferentially form anisotropic systems with relatively high ellipticities, not consistent with observed isotropic and round ellipticals. This is kinematical evidence that some ellipticals cannot have formed by mergers of disks in the first place. In addition alternative formation scenarios are motivated based on stellar population considerations (Naab \& Ostriker 2007) as well as direct cosmological simulations (Naab et al. 2007).

AGNs are emerging as an important ingredient in our current galaxy formation models. Research on understanding the observed $M_{\mathrm{BH}}-\sigma, M_{\mathrm{BH}}-M_{*}$ and $M_{\mathrm{BH}}-M_{*} \sigma^{2}$ relations using numerical techniques should therefore be a useful step in our quest to formulate a more complete theory of galaxy formation.

We would like to thank the anonymous referee for a careful reading of the manuscript and valuable comments. In addition we thank V. Springel for helpful comments and for supplying data for a comparison simulation. Furthermore, we thank P. Hopkins, R. Jesseit, J. Sommer-Larsen and M. Wetzstein for stimulating discussions and helpful comments on the manuscript. The numerical simulations were performed on the local SGIAltix $3700 \mathrm{Bx} 2$, which was partly funded by the Cluster 
of Excellence: "Origin and Structure of the Universe".

\section{REFERENCES}

Abadi, M. G., Navarro, J. F., Steinmetz, M., \& Eke, V. R. 2003, ApJ, 597, 21

Adams, F. C., Graff, D. S., \& Richstone, D. O. 2001, ApJ, 551, L31

Aller, M. C. \& Richstone, D. O. 2007, ApJ, 665, 120

Arad, I. \& Johansson, P. H. 2005, MNRAS, 362, 252

Barger, A. J., Cowie, L. L., Mushotzky, R. F., Yang, Y., Wang, W.-H., Steffen, A. T., \& Capak, P. 2005, AJ, 129, 578

Barnes, J. E. 1998, in Saas-Fee Advanced Course 26: Galaxies: Interactions and Induced Star Formation, ed. R. C. Kennicutt, Jr., F. Schweizer, J. E. Barnes, D. Friedli, L. Martinet, \& D. Pfenniger, 275-+

Barnes, J. E. 2002, MNRAS, 333, 481

Barnes, J. E. \& Hernquist, L. 1996, ApJ, 471, 115

Barway, S. \& Kembhavi, A. 2007, ApJ, 662, L67

Bate, M. R. \& Burkert, A. 1997, MNRAS, 288, 1060

Bell, E. F., Naab, T., McIntosh, D. H., Somerville, R. S., Caldwell, J. A. R., Barden, M., Wolf, C., Rix, H.-W., Beckwith, S. V., Borch, A., Häussler, B., Heymans, C., Jahnke, K., Jogee, S., Koposov, S., Meisenheimer, K., Peng, C. Y., Sanchez, S. F., \& Wisotzki, L. 2006, ApJ, 640, 241

Bondi, H. 1952, MNRAS, 112, 195

Bondi, H. \& Hoyle, F. 1944, MNRAS, 104, 273

Bournaud, F., Elmegreen, B. G., \& Elmegreen, D. M. 2007a, ApJ, 670, 237

Bournaud, F., Jog, C. J., \& Combes, F. 2005, A\&A, 437, 69

-. 2007b, A\&A, 476, 1179

Bower, R. G., Benson, A. J., Malbon, R., Helly, J. C., Frenk, C. S., Baugh, C. M., Cole, S., \& Lacey, C. G. 2006, MNRAS, 370, 645

Boylan-Kolchin, M., Ma, C.-P., \& Quataert, E. 2005, MNRAS, 362, 184

Bullock, J. S., Kolatt, T. S., Sigad, Y., Somerville, R. S., Kravtsov, A. V., Klypin, A. A., Primack, J. R., \& Dekel, A. 2001, MNRAS, 321,559

Burkert, A. \& Naab, T. 2005, MNRAS, 363, 597

Burkert, A., Naab, T., \& Johansson, P. H. 2007, ArXiv e-prints, 710, arXiv:0710.0663

Burkert, A. \& Silk, J. 2001, ApJ, 554, L151

Canalizo, G. \& Stockton, A. 2001, ApJ, 555, 719

Cappellari, M., Bacon, R., Bureau, M., Damen, M. C., Davies, R. L., de Zeeuw, P. T., Emsellem, E., Falcón-Barroso, J., Krajnović, D., Kuntschner, H., McDermid, R. M., Peletier, R. F., Sarzi, M., van den Bosch, R. C. E., \& van de Ven, G. 2006, MNRAS, 366, 1126

Cattaneo, A., Haehnelt, M. G., \& Rees, M. J. 1999, MNRAS, 308, 77

Ciotti, L. \& Ostriker, J. P. 1997, ApJ, 487, L105+

-. 2007, ApJ, 665, 1038

Ciotti, L. \& van Albada, T. S. 2001, ApJ, 552, L13

Cox, T. J., Dutta, S. N., Di Matteo, T., Hernquist, L., Hopkins, P. F., Robertson, B., \& Springel, V. 2006, ApJ, 650, 791

Cox, T. J., Jonsson, P., Somerville, R. S., Primack, J. R., \& Dekel, A. 2008, MNRAS, 33

Cretton, N., Naab, T., Rix, H.-W., \& Burkert, A. 2001, ApJ, 554, 291

Croton, D. J., Springel, V., White, S. D. M., De Lucia, G., Frenk, C. S., Gao, L., Jenkins, A., Kauffmann, G., Navarro, J. F., \& Yoshida, N. 2006, MNRAS, 365, 11

Daddi, E., Alexander, D. M., Dickinson, M., Gilli, R., Renzini, A., Elbaz, D., Cimatti, A., Chary, R., Frayer, D., Bauer, F. E., Brandt, W. N., Giavalisco, M., Grogin, N. A., Huynh, M., Kurk, J., Mignoli, M., Morrison, G., Pope, A., \& Ravindranath, S. 2007a, ApJ, 670, 173

Daddi, E., Dickinson, M., Morrison, G., Chary, R., Cimatti, A., Elbaz, D., Frayer, D., Renzini, A., Pope, A., Alexander, D. M., Bauer, F. E., Giavalisco, M., Huynh, M., Kurk, J., \& Mignoli, M. 2007b, ApJ, 670, 156

Dasyra, K. M., Tacconi, L. J., Davies, R. I., Genzel, R., Lutz, D., Naab, T., Burkert, A., Veilleux, S., \& Sanders, D. B. 2006a, ApJ, 638,745

Dasyra, K. M., Tacconi, L. J., Davies, R. I., Genzel, R., Lutz, D., Naab, T., Sanders, D. B., Veilleux, S., \& Baker, A. J. 2006b, New Astronomy Review, 50, 720
Dasyra, K. M., Tacconi, L. J., Davies, R. I., Naab, T., Genzel, R., Lutz, D., Sturm, E., Baker, A. J., Veilleux, S., Sanders, D. B., \& Burkert, A. 2006c, ApJ, 651, 835

Dehnen, W. 2001, MNRAS, 324, 273

di Matteo, P., Combes, F., Melchior, A.-L., \& Semelin, B. 2007, A\&A, 468, 61

Di Matteo, T., Colberg, J., Springel, V., Hernquist, L., \& Sijacki, D. 2008, ApJ, 676, 33

Di Matteo, T., Springel, V., \& Hernquist, L. 2005, Nature, 433, 604

Efstathiou, G. 2000, MNRAS, 317, 697

Elmegreen, B. G., Bournaud, F., \& Elmegreen, D. M. 2008, ArXiv e-prints, 805, arXiv:0805.2266

Emsellem, E., Cappellari, M., Peletier, R. F., McDermid, R. M., Bacon, R., Bureau, M., Copin, Y., Davies, R. L., Krajnović, D., Kuntschner, H., Miller, B. W., \& de Zeeuw, P. T. 2004, MNRAS, 352,721

Escala, A., Larson, R. B., Coppi, P. S., \& Mardones, D. 2004, ApJ, 607,765

Fabian, A. C. 1999, MNRAS, 308, L39

Ferrarese, L. \& Merritt, D. 2000, ApJ, 539, L9

Flanagan, É. É. \& Hughes, S. A. 1998, Phys. Rev. D, 57, 4535

Gebhardt, K., Bender, R., Bower, G., Dressler, A., Faber, S. M., Filippenko, A. V., Green, R., Grillmair, C., Ho, L. C., Kormendy, J., Lauer, T. R., Magorrian, J., Pinkney, J., Richstone, D., \& Tremaine, S. 2000, ApJ, 539, L13

Genzel, R., Lutz, D., Sturm, E., Egami, E., Kunze, D., Moorwood, A. F. M., Rigopoulou, D., Spoon, H. W. W., Sternberg, A., Tacconi-Garman, L. E., Tacconi, L., \& Thatte, N. 1998, ApJ, 498,579

Genzel, R., Tacconi, L. J., Rigopoulou, D., Lutz, D., \& Tecza, M. 2001, ApJ, 563, 527

Granato, G. L., De Zotti, G., Silva, L., Bressan, A., \& Danese, L. 2004, ApJ, 600, 580

Haardt, F. \& Madau, P. 1996, ApJ, 461, 20

Häring, N. \& Rix, H.-W. 2004, ApJ, 604, L89

Hernquist, L. 1989, Nature, 340, 687

-. 1990, ApJ, 356, 359

Hibbard, J. E. \& Yun, M. S. 1999, ApJ, 522, L93

Hopkins, P. F., Cox, T. J., Kereš, D., \& Hernquist, L. 2008a, ApJS, 175,390

Hopkins, P. F., Hernquist, L., Cox, T. J., Di Matteo, T., Martini, P., Robertson, B., \& Springel, V. 2005, ApJ, 630, 705

Hopkins, P. F., Hernquist, L., Cox, T. J., Di Matteo, T., Robertson, B., \& Springel, V. 2006, ApJS, 163, 1

Hopkins, P. F., Hernquist, L., Cox, T. J., \& Kereš, D. 2008b, ApJS, 175,356

Hopkins, P. F., Hernquist, L., Cox, T. J., Robertson, B., \& Krause, E. 2007a, ApJ, 669, 45

- 2007b, ApJ, 669, 67

Houck, J. R., Soifer, B. T., Weedman, D., Higdon, S. J. U., Higdon, J. L., Herter, T., Brown, M. J. I., Dey, A., Jannuzi, B. T., Le Floc'h, E., Rieke, M., Armus, L., Charmandaris, V., Brandl, B. R., \& Teplitz, H. I. 2005, ApJ, 622, L105

Hoyle, F. \& Lyttleton, R. A. 1939, in Proceedings of the Cambridge Philisophical Society, Vol. 34, Proceedings of the Cambridge Philisophical Society, 405--

Hughes, S. A. 2002, MNRAS, 331, 805

Jesseit, R., Naab, T., \& Burkert, A. 2005, MNRAS, 360, 1185

Jesseit, R., Naab, T., Peletier, R. F., \& Burkert, A. 2007, MNRAS, 376,997

Johansson, P. H. \& Efstathiou, G. 2006, MNRAS, 371, 1519

Johansson, P. H., Väisänen, P., \& Vaccari, M. 2004, A\&A, 427, 795

Katz, N., Weinberg, D. H., \& Hernquist, L. 1996, ApJS, 105, 19

Kauffmann, G. \& Haehnelt, M. 2000, MNRAS, 311, 576

Kazantzidis, S., Mayer, L., Colpi, M., Madau, P., Debattista, V. P., Wadsley, J., Stadel, J., Quinn, T., \& Moore, B. 2005, ApJ, 623, L67

Kennicutt, Jr., R. C. 1998, ARA\&A, 36, 189

Khalatyan, A., Cattaneo, A., Schramm, M., Gottlöber, S.,

Steinmetz, M., \& Wisotzki, L. 2008, MNRAS, 387, 13

Khochfar, S. \& Burkert, A. 2001, ApJ, 561, 517

-. 2003, ApJ, 597, L117

-. 2005, MNRAS, 359, 1379 
-. 2006, A\&A, 445, 403

Khochfar, S. \& Silk, J. 2006, MNRAS, 370, 902

Kormendy, J. \& Richstone, D. 1995, ARA\&A, 33, 581

Levine, R., Gnedin, N. Y., Hamilton, A. J. S., \& Kravtsov, A. V. 2008, ApJ, 678, 154

Lynden-Bell, D. 1967, MNRAS, 136, 101

Madau, P. \& Quataert, E. 2004, ApJ, 606, L17

Magorrian, J., Tremaine, S., Richstone, D., Bender, R., Bower, G., Dressler, A., Faber, S. M., Gebhardt, K., Green, R., Grillmair, C., Kormendy, J., \& Lauer, T. 1998, AJ, 115, 2285

Makino, J. \& Funato, Y. 2004, ApJ, 602, 93

Marconi, A. \& Hunt, L. K. 2003, ApJ, 589, L21

Mayer, L., Kazantzidis, S., Madau, P., Colpi, M., Quinn, T., \& Wadsley, J. 2007, Science, 316, 1874

McKee, C. F. \& Ostriker, J. P. 1977, ApJ, 218, 148

McLure, R. J., Kukula, M. J., Dunlop, J. S., Baum, S. A., O’Dea, C. P., \& Hughes, D. H. 1999, MNRAS, 308, 377

Merritt, D. \& Milosavljević, M. 2005, Living Reviews in Relativity, 8,8

Merritt, D., Milosavljević, M., Favata, M., Hughes, S. A., \& Holz, D. E. 2004, ApJ, 607, L9

Mo, H. J., Mao, S., \& White, S. D. M. 1998, MNRAS, 295, 319

Monaghan, J. J. 1992, ARA\&A, 30, 543

Murray, N., Quataert, E., \& Thompson, T. A. 2005, ApJ, 618, 569

Naab, T. \& Burkert, A. 2003, ApJ, 597, 893

Naab, T., Burkert, A., \& Hernquist, L. 1999, ApJ, 523, L133

Naab, T., Jesseit, R., \& Burkert, A. 2006a, MNRAS, 372, 839

Naab, T., Johansson, P. H., Ostriker, J. P., \& Efstathiou, G. 2007, ApJ, 658, 710

Naab, T., Khochfar, S., \& Burkert, A. 2006b, ApJ, 636, L81

Naab, T. \& Ostriker, J. P. 2007, ArXiv Astrophysics e-prints, 702, 0702535

Naab, T. \& Trujillo, I. 2006, MNRAS, 369, 625

Navarro, J. F., Frenk, C. S., \& White, S. D. M. 1997, ApJ, 490, 493

Nipoti, C., Londrillo, P., \& Ciotti, L. 2003, MNRAS, 342, 501

Oppenheimer, B. D. \& Davé, R. 2006, MNRAS, 373, 1265

Richstone, D., Ajhar, E. A., Bender, R., Bower, G., Dressler, A., Faber, S. M., Filippenko, A. V., Gebhardt, K., Green, R., Ho, L. C., Kormendy, J., Lauer, T. R., Magorrian, J., \& Tremaine, S. 1998, Nature, 395, A14+

Robertson, B., Bullock, J. S., Cox, T. J., Di Matteo, T., Hernquist, L., Springel, V., \& Yoshida, N. 2006a, ApJ, 645, 986
Robertson, B., Cox, T. J., Hernquist, L., Franx, M., Hopkins, P. F., Martini, P., \& Springel, V. 2006b, ApJ, 641, 21

Robertson, B., Hernquist, L., Cox, T. J., Di Matteo, T., Hopkins,

P. F., Martini, P., \& Springel, V. 2006c, ApJ, 641, 90

Rothberg, B. \& Joseph, R. D. 2006, AJ, 132, 976

Saslaw, W. C., Valtonen, M. J., \& Aarseth, S. J. 1974, ApJ, 190, 253

Shakura, N. I. \& Syunyaev, R. A. 1973, A\&A, 24, 337

Sijacki, D. \& Springel, V. 2006, MNRAS, 366, 397

Sijacki, D., Springel, V., di Matteo, T., \& Hernquist, L. 2007, MNRAS, 380, 877

Silk, J. \& Rees, M. J. 1998, A\&A, 331, L1

Sillanpää, A., Haarala, S., Valtonen, M. J., Sundelius, B., \& Byrd, G. G. 1988, ApJ, 325, 628

Springel, V. 2005, MNRAS, 364, 1105

Springel, V., Di Matteo, T., \& Hernquist, L. 2005a, ApJ, 620, L79

-. 2005b, MNRAS, 361, 776

Springel, V. \& Hernquist, L. 2002, MNRAS, 333, 649

-. 2003, MNRAS, 339, 289

-. 2005, ApJ, 622, L9

Thomas, J., Jesseit, R., Naab, T., Saglia, R. P., Burkert, A., \& Bender, R. 2007, MNRAS, 381, 1672

Tran, K.-V. H., van Dokkum, P., Franx, M., Illingworth, G. D., Kelson, D. D., \& Schreiber, N. M. F. 2005, ApJ, 627, L25

Tremaine, S., Gebhardt, K., Bender, R., Bower, G., Dressler, A., Faber, S. M., Filippenko, A. V., Green, R., Grillmair, C., Ho, L. C., Kormendy, J., Lauer, T. R., Magorrian, J., Pinkney, J., \& Richstone, D. 2002, ApJ, 574, 740

Väisänen, P., Mattila, S., Kniazev, A., Adamo, A., Efstathiou, A., Farrah, D., Johansson, P. H., Östlin, G., Buckley, D. A. H., Burgh, E. B., Crause, L., Hashimoto, Y., Lira, P., Loaring, N., Nordsieck, K., Romero-Colmenero, E., Ryder, S., Still, M., \& Zijlstra, A. 2008, MNRAS, 110

van Dokkum, P. G. 2005, AJ, 130, 2647

van Dokkum, P. G., Franx, M., Fabricant, D., Kelson, D. D., \& Illingworth, G. D. 1999, ApJ, 520, L95

Vitvitska, M., Klypin, A. A., Kravtsov, A. V., Wechsler, R. H., Primack, J. R., \& Bullock, J. S. 2002, ApJ, 581, 799

Volonteri, M., Haardt, F., \& Madau, P. 2003, ApJ, 582, 559

Wyithe, J. S. B. \& Loeb, A. 2003, ApJ, 595, 614

\section{APPENDIX}

\section{A. BLACK HOLE FEEDBACK MODEL}

The growth of the supermassive black holes is modeled following the Springel et al. (2005b) model. In this effective subresolution model the unresolved accretion onto the $\mathrm{BH}$ is related to the resolved gas distribution using a BondiHoyle-Lyttleton parameterization (Hoyle \& Lyttleton 1939; Bondi \& Hoyle 1944; Bondi 1952). In this description, the accretion rate onto the $\mathrm{BH}$ is given by

$$
\dot{M}_{\mathrm{B}}=\frac{4 \pi \alpha G^{2} M_{\mathrm{BH}}^{2} \rho}{\left(c_{\mathrm{s}}^{2}+v^{2}\right)^{3 / 2}} .
$$

Here $\rho$ and $c_{\mathrm{S}}$ are the density and sound speed of the surrounding gas, respectively, $v$ is the velocity of the BH relative to the surrounding gas and $\alpha$ is a dimensionless parameter setting the efficiency of the accretion. In addition, it is assumed that the maximum accretion is limited to the Eddington rate

$$
\dot{M}_{\text {edd }}=\frac{4 \pi G M_{\mathrm{BH}} m_{\mathrm{p}}}{\epsilon_{\mathrm{r}} \sigma_{\mathrm{T}} c}
$$

where $m_{\mathrm{p}}$ is the proton mass, $\sigma_{\mathrm{T}}$ is the Thomson cross-section and $\epsilon_{\mathrm{r}}$ is the radiative efficiency, that we assume to be 0.1 based on the mean value for radiatively efficient Shakura \& Syunyaev (1973) accretion onto a Schwarzschild BH. Thus the accretion rate is given by $\dot{M}_{\mathrm{BH}}=\min \left(\dot{\mathrm{M}}_{\mathrm{B}}, \dot{\mathrm{M}}_{\mathrm{edd}}\right)$. Finally some fraction $\epsilon_{\mathrm{f}}$ of the radiated luminosity $L_{\mathrm{r}}$ couples thermally and isotropically to the surrounding gas in the form of black hole feedback energy resulting in

$$
\dot{E}_{\mathrm{feed}}=\epsilon_{f} L_{\mathrm{r}}=\epsilon_{f} \epsilon_{\mathrm{r}} \dot{M}_{\mathrm{BH}} c^{2}
$$

Again, following Springel et al. (2005b), we fix $\epsilon_{f}=0.05$ so that in total 0.5 per cent of the accreted rest mass energy is available as feedback. This choice fixes the normalization of the $M_{\mathrm{BH}}-\sigma$ relation and reproduces the observed relation in equal-mass binary galaxy merger simulations with $\mathrm{BH}$ feedback as was shown by Di Matteo et al. (2005). The feedback energy is distributed as thermal energy to the surrounding gas particles weighted with the SPH kernel. 


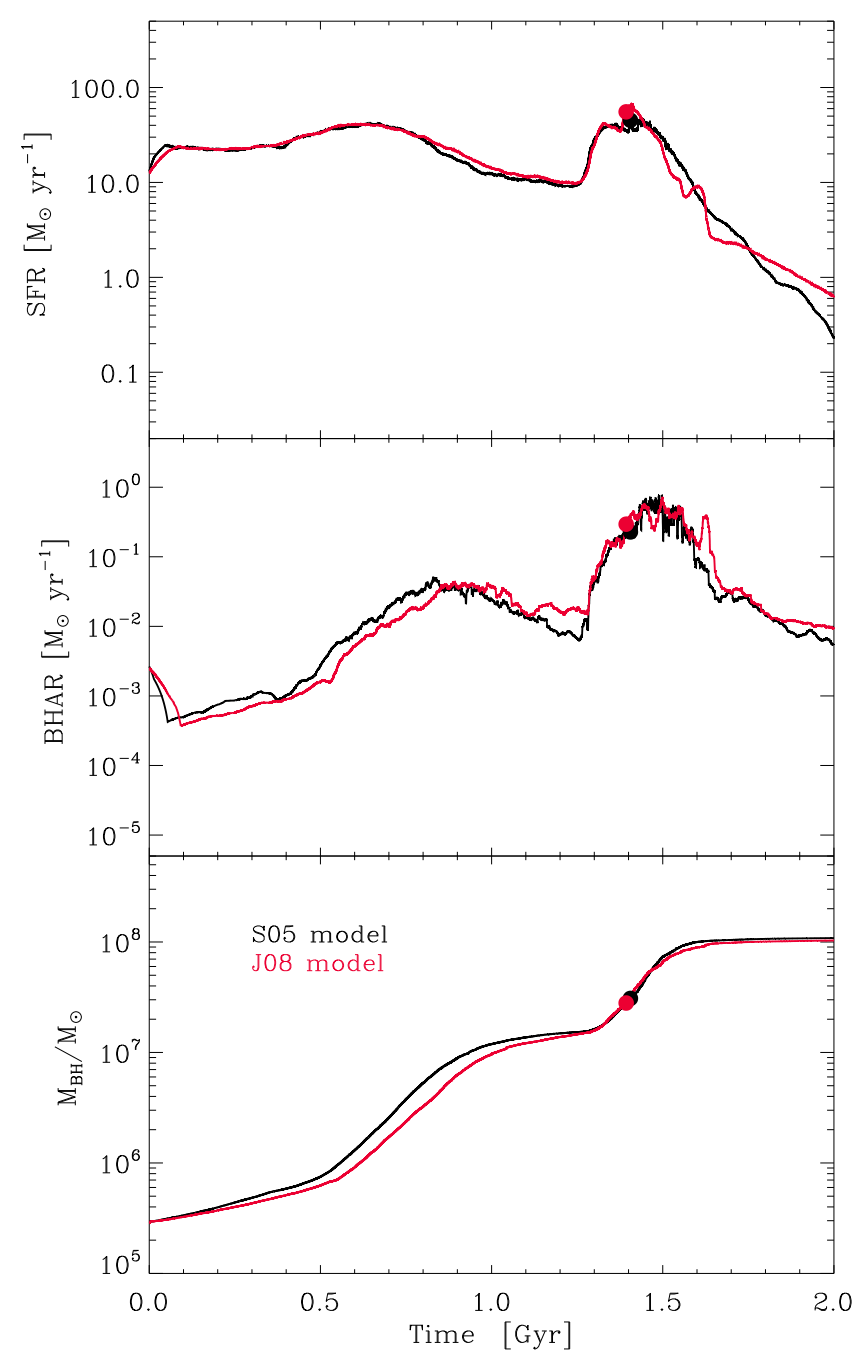

FIG. B1.- Comparison of the total star formation rate (top), the total black hole accretion rate (middle) and the evolution of the total black hole mass (bottom) as a function of time for a 1:1 merger run using the Springel et al. (2005b) BH feedback S05 model and the model presented in this paper (J08 model). The filled circles indicate the time of merging of the BHs.

Numerically the BHs are represented as collisionless 'sink' particles, which only feel gravitational forces. The properties, such as density, temperature and the bulk velocity of the local gas around the $\mathrm{BH}$ are estimated in a similar fashion to normal SPH particles. Based on these quantities and the equations above, the BH accretion rate is then estimated.

\section{B. COMPARISON OF THE BH FEEDBACK MODELS}

We perform a direct comparison of our BH feedback model to the Springel et al. (2005b) model by running one of the initial conditions used in the Springel et al. (2005b) study (kindly provided by Volker Springel) using our simulation code. We use identical numerical parameters governing the stellar feedback, BH feedback and integration accuracy. In Fig. B1 we plot the resulting star formation rate, $\mathrm{BH}$ accretion rate and total $\mathrm{BH}$ mass for the simulation performed using the Springel et al. (2005b) (S05 model, data provided by Volker Springel) and compare it to the corresponding output of the model presented in this paper (J08 model).

The agreement between the two codes is very good, especially given the fact that both the star formation and $\mathrm{BH}$ accretion processes are treated stochastically. The star formation histories agree very well up to the time of the merging of the BHs. After the merging of the BHs we see small differences in the decay rate of star formation in the two models. This slight discrepancy is due to the differences in the corresponding BH accretion rates resulting in the final BH being marginally more massive in the S05 model. The late decay of star formation is relatively sensitive to the final $\mathrm{BH}$ mass, with more massive BHs resulting in more efficient termination of star formation and hence small differences in the late star formation history are not unexpected.

We thus conclude given the stochastic nature of the modeled processes that the resulting agreement on the $10 \%$ level between the two codes is very good. Thus we expect our BH feedback prescription to produce nearly identical results compared to the original Springel et al. (2005b) model. 

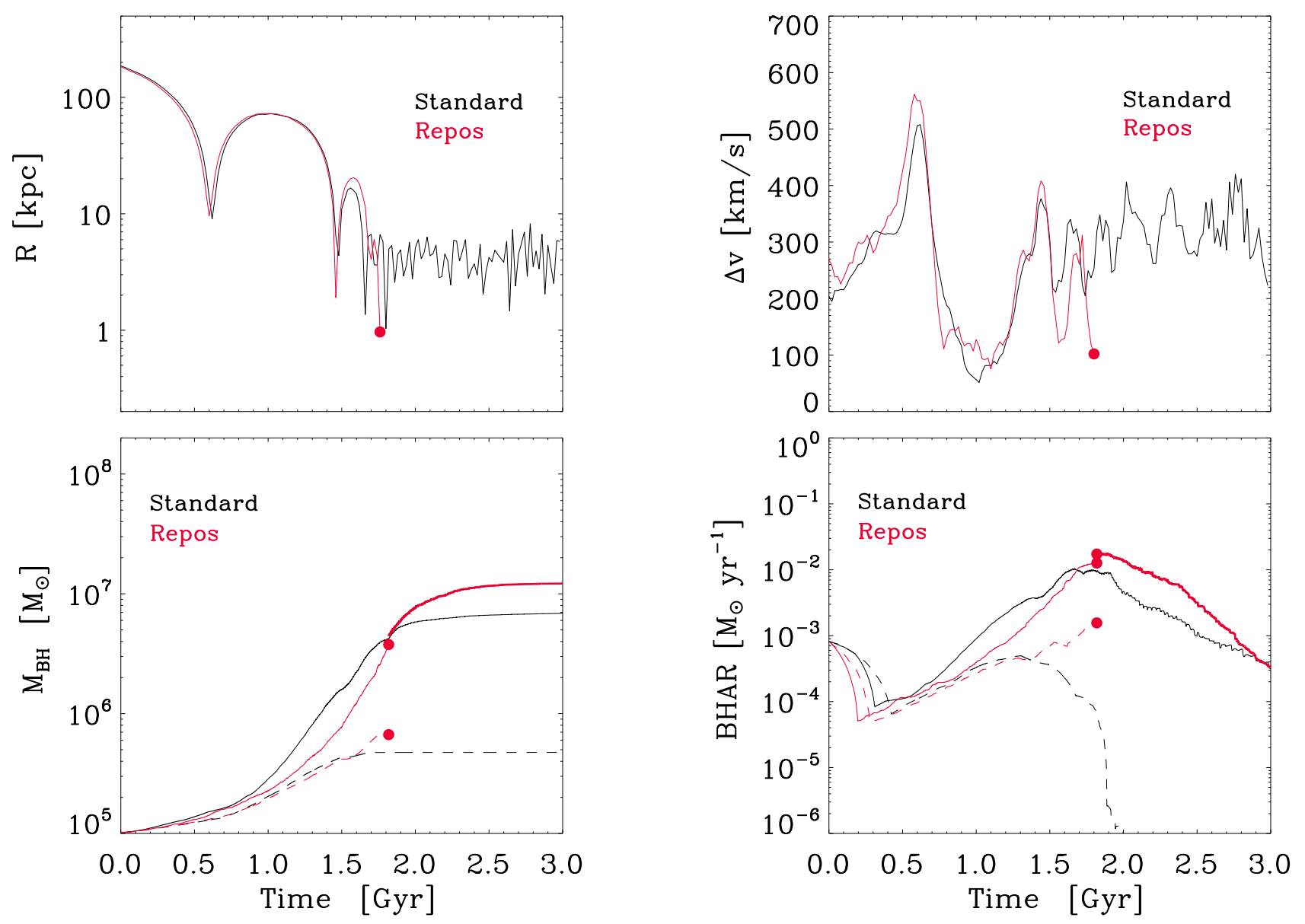

FIG. C1. - 3:1 unequal-mass merger simulations run at the standard numerical resolution with the standard BH merger prescription (black lines) and the repositioning method (red lines). The top left and right panels shows the relative distances and velocities between the $\mathrm{BHs}$ as a function of time. The bottom panels show the evolution of the $\mathrm{BH}$ masses and accretion rates as a function of time. The solid line depicts the primary $\mathrm{BH}$, the dashed line the secondary $\mathrm{BH}$ and the thick solid line the merged $\mathrm{BH}$, with the time of merging indicated with solid circles.

\section{COMPARISON OF THE MERGER PRESCRIPTIONS}

We plot in Fig. C1 the black hole mass growth and mass accretion rate together with the distance and relative velocity between the black holes for one unequal-mass 3:1 merger at our standard resolution using both the standard and repositioning merger prescriptions. The galaxies go through their first and second passages at $t_{1}=0.6 \mathrm{Gyr}$ and $t_{2}=1.4 \mathrm{Gyr}$ respectively. The galaxies then approach each other for a third time at $t_{3}=1.8 \mathrm{Gyr}$ at which time the $\mathrm{BH}$ in the simulation with $\mathrm{BH}$ repositioning merge, unlike the standard case in which the BHs do not merge. The mass growth of both the primary (solid lines) and the secondary (dashed lines) BHs are similar for both methods until the merger, after which time essentially only the repositioned BH grows in mass. A similar result can be seen in the $\mathrm{BH}$ accretion rates, which are similar until $t_{3}=1.8 \mathrm{Gyr}$, after which the repositioned BH shows almost an order of magnitude higher accretion rates compared to the standard BH merger case. One can also see that the secondary $\mathrm{BH}$ mass accretion rate dramatically drops at this time, hence terminating the growth of the secondary BH. This is caused by the fact that the secondary BH is perturbed away from its surrounding gas disk during the final stages of the merger.

In the standard $\mathrm{BH}$ merger prescription the merging of the $\mathrm{BH}$ s is typically delayed with respect to the $\mathrm{BH}$ in the repositioning runs. During the $\mathrm{BH}$ merger the momentum is conserved, which can give the $\mathrm{BH}$ a relatively large 'kick', especially in the 3:1 mergers where the mass ratio of the BHs is relatively large. The repositioning ensures that after the merger the BH quickly resettles in the center of the central gas disk that is feeding the BH. In the standard prescription the merged $\mathrm{BH}$ remains longer displaced from the central gas disk, thus lowering the mass accretion rate, which scales linearly with density $\left(\dot{M}_{\mathrm{B}} \propto \rho\right.$ in Eq. A1). The repositioning also lowers the relative velocity of the BH with respect to the surrounding gas. For the standard merging case the relative velocity can be relatively large with the $\mathrm{BH}$ plunging through the central gaseous disk. This also affects the $\mathrm{BH}$ accretion rate as it scales as the inverse third power of the relative BH-gas velocity for a fixed sound speed of the medium $\left(\dot{M}_{\mathrm{B}} \propto v^{-3}\right.$ in Eq. A1). Finally the $\mathrm{BH}$ accretion rate scales with the square of the BH mass $\left(\dot{M}_{\mathrm{B}} \propto M_{\mathrm{BH}}^{2}\right.$ in Eq. A1) resulting in higher accretion rates for more massive BHs, thus an earlier merging of the BHs produces typically larger final $\mathrm{BH}$ masses.

In Fig. C2 we plot the corresponding details for a 1:1 merger at our standard numerical resolution using the 

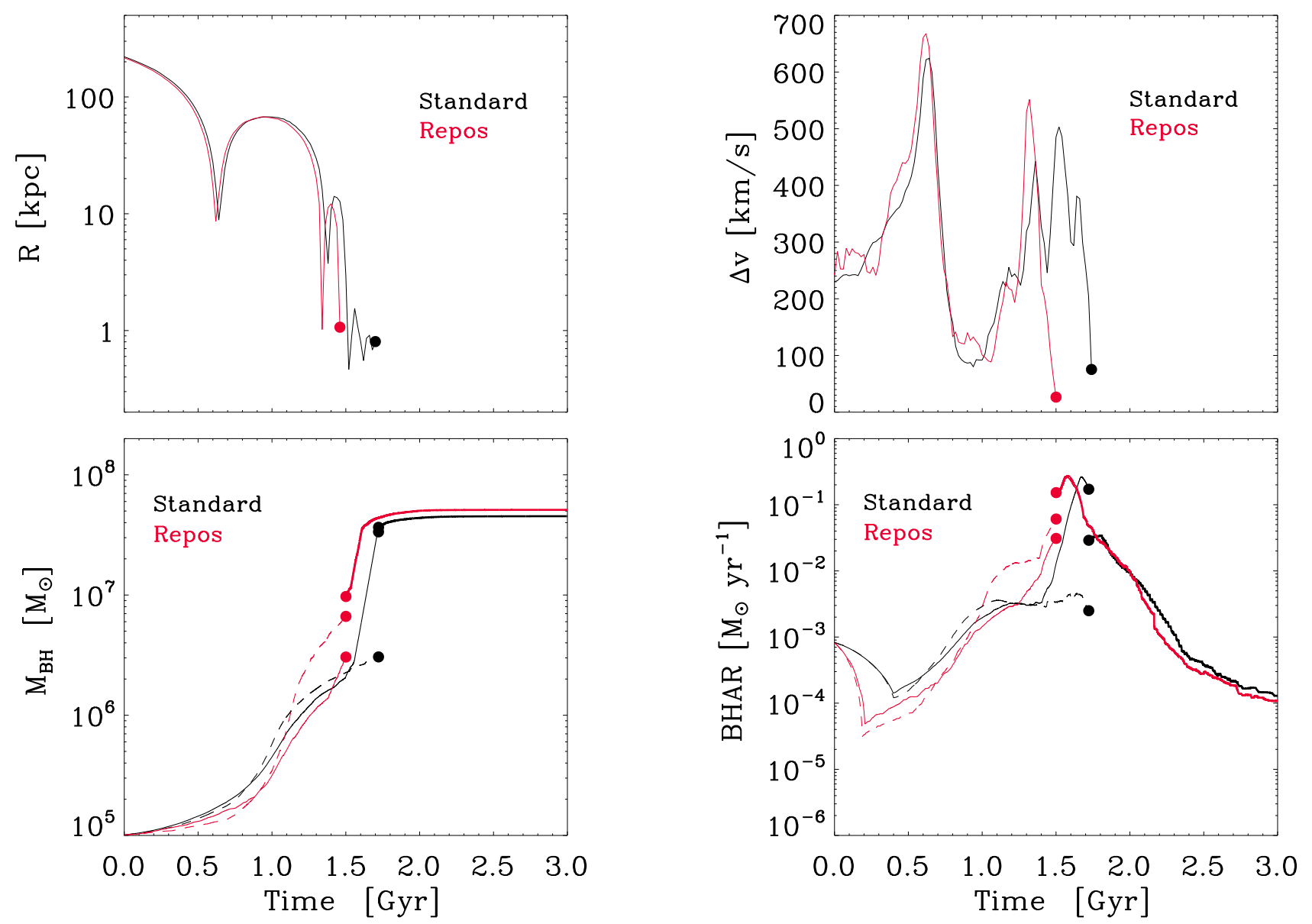

FIG. C2.- 1:1 equal-mass merger simulations run at the standard numerical resolution with the standard BH merger prescription (black lines) and the repositioning method (red lines). The top left and right panels shows the relative distances and velocities between the BHs as a function of time. The bottom panels show the evolution of the BH masses and accretion rates as a function of time. The solid line depicts the primary $\mathrm{BH}$, the dashed line the secondary $\mathrm{BH}$ and the thick solid line the merged $\mathrm{BH}$, with the time of merging indicated with solid circles.

two merging prescriptions. The differences between the merger prescriptions compared to the 3:1 merger are less pronounced with the BHs merging slightly earlier in the repositioned simulation. However, the growth of the black hole masses show differences with the $\mathrm{BHs}$ in the repositioned run showing roughly equal mass growth prior to the $\mathrm{BH}$ merging compared to the standard case, where the more massive $\mathrm{BH}$ is an order of magnitude more massive than the secondary $\mathrm{BH}$ at the time of the $\mathrm{BH}$ merger. In the standard merging case the secondary $\mathrm{BH}$ is perturbed away from its gaseous central disk prior to the final merger, thus lowering the accretion rate and growth of the $\mathrm{BH}$ in comparison to the primary $\mathrm{BH}$. After the merger the repositioned $\mathrm{BH}$ exhibits strong growth in a gas-rich environment until the thermal feedback energy input is large enough to clear away the remaining gas, thus terminating the $\mathrm{BH}$ growth. This is not mirrored in the standard merging case, where the primary BH grows strongly prior to merging and only modestly after the merger due to the fact that the merged $\mathrm{BH}$ is unable to resettle in the remaining central gas disk. However, the end result of both merging prescriptions is a final $\mathrm{BH}$ mass and stellar velocity dispersion that agree with each other on the $10 \%$ level.

Taken together these results indicate that repositioning the BHs produce converged and stable results for the mass growth and accretion rates of the BHs. Repositioning is essential in representing the evolution of BHs in 3:1 mergers and also produces converged results for 1:1 mergers even at relatively low numerical resolution. 